\title{
A New Intelligent Weak Fault Recognition Framework for Rotating Machinery
}

\author{
Xiaoli Zhao \\ School of Mechanical Engineering, Southeast University, Nanjing 211189, China. \\ School of Engineering, University of British Columbia, Kelowna, BC VIV 1V7, Canada. \\ Minping Jia, Peng Ding, Cheng Yang and Daoming She \\ School of Mechanical Engineering, Southeast University, Nanjing 211189, China.
}

Lin Zhu

School of Mechanical Engineering, Yangzhou University, Yangzhou 225001, China.

\author{
Zheng Liu \\ School of Engineering, University of British Columbia, Kelowna, BC V1V 1V7, Canada.
}

(Received 19 March 2020; accepted 13 July 2020)

\begin{abstract}
The presence of strong background noises makes it a challenging task to detect weak fault characteristics in vibration signals collected from rotating machinery. Thus, a two-stage intelligent weak fault recognition framework, which includes signal enhancement and intelligent recognition, is proposed in this work. The signal enhancement is accomplished via an optimized relevant variational mode decomposition (ORVMD) algorithm. Specifically, the optimal parameters is derived by combining a particle swarm optimization (PSO) algorithm and the novel defined relevant energy (Re) index. This optimized VMD algorithm can extract the principal components from the raw signals. Then, the enhanced vibration signals via the proposed ORVMD are converted into spectral signals and fed into an improved stacked auto-encoder (ISAE) algorithm for fault recognition. Experimental results demonstrate the effectiveness of the proposed algorithms and fault diagnosis framework in rotating machinery fault recognition and detection.
\end{abstract}

\section{INTRODUCTION}

Large-scale rotating machinery, such as steam turbines and wind turbines, are widely used in current industrial fields. ${ }^{1-3}$ They play a vital role in economic production and industrial manufacturing. However, rotating machinery is prone to failure, which could damage property and human life., ${ }^{4,5}$ Early detection or diagnosis of the potential weak fault in these systems is desirable to cut down the economic loss of industrial production and increase the benefits. ${ }^{6,7}$

The vibration signals captured from rotating machinery are mostly non-stationary and consist of multiple-component aliased vibration signals. These signals are usually affected by the attenuation of the vibration in the transmission path and background noise. These signal characteristics seriously impede the fault information extraction from the raw industrial vibration signals. ${ }^{8}$ Compared with the severe or late-in-life faults of rotating machinery, it is a more challenging topic to extract and recognize weak fault feature information in advance. ${ }^{8-10}$

Moreover, the multiple-component vibration signals are separated into many single component signals from each vibration source. If the fault features can be effectively extracted from the noises, fault diagnosis techniques become more effective.

The weak fault diagnosis method based on time-frequency analysis is widely used in the field of fault diagnosis and detection of rotating machinery. It is mainly divided into two modes: one is recursive signal decomposition, and the other is non-recursive signal decomposition. ${ }^{8-11}$ For the recursive signal decomposition, Huang et al. proposed an adaptive signal processing method called empirical mode decomposition (EMD). ${ }^{11}$ In the EMD, a given multiple-component signal is decomposed into a series of components under different frequency bands. ${ }^{11,12}$ However, there still exist some problems, such as mode mixing, which will result in obstacles on signal decomposition and reconstruction. ${ }^{12,13}$ Inspired from EMD, Smith et al. proposed a new time-frequency signal decomposition method, namely local mean decomposition (LMD), which can adaptively decompose a given nonstationary signal into a linear combination of multiple product function (PF) components. ${ }^{14} \mathrm{Su}$ et al. developed an early fault diagnosis method based on EMD and spectral kurtosis. ${ }^{15}$ Bin et al. constructed a feature extraction approach built on the combination of wavelet packet decomposition (WPD) and EMD. ${ }^{16} \mathrm{Li}$ et al. proposed an LMD algorithm based on differential rational splines (DRSLMD) for early fault diagnosis in rotating machinery. ${ }^{17}$ At the same time, Kullback-Leibler (K-L) divergence was applied to select the sensitive product function (PF) component signals. Due to the interference of background noise and amplitude modulation effects, the weak faults in planetary gear systems are difficult to detect. To detect these weak faults in planetary gearboxes, a comprehensive diagnostic method called improved maximum correlation kurtosis deconvolution (IMCKD) was proposed by Zhang. ${ }^{5}$ The weak root crack in the single-stage planetary sun gear was used to prove its effectiveness. Cheng et al. proposed an improved symplectic ge- 
ometric mode decomposition (ISGMD) that contains the vast majority of fault feature information for the early processing of gear fault signals. ${ }^{8}$ Simulation and experimental results suggest that ISMGD is effective for early fault signal decomposition. To reveal the dynamic characteristics of the rubbing fault signal of a rotor system, Yu et al. proposed a second-order multi-synchronous compression algorithm, which embedded the second-order two-dimensional instantaneous frequency estimation into multi-synchronous compression. ${ }^{10}$

The signal decomposition algorithms are all recursive mode. ${ }^{18-20}$ For the recursive signal decomposition, Dragomiretskiy developed an adaptive signal decomposition algorithm known as variational mode decomposition (VMD). ${ }^{21}$ In this method the component decomposition is iterative and an optimal search is performed to determine the center frequency and bandwidth of each component. Compared with other existing recursive "filtering" mode methods, the VMD algorithm has a solid mathematical and theoretical basis. Hence, the given signals are decomposed more accurately via VMD. ${ }^{21,22}$

Currently, VMD has been widely applied in fault diagnosis, and has demonstrated its capability in signal decomposition and feature extraction. ${ }^{18,22-24}$ Mohanty et al. utilized the advantage of VMD to decompose vibration signals of the rolling bearing into a series of IMF components signals from which the different health conditions of rolling bearing signals were recognized. ${ }^{25}$ And Yang showed the noise signal of hydro power units was decomposed via VMD, then the decomposed signal was naturally reconstructed. ${ }^{26}$ Wang et al. used VMD to successfully recognize the different friction and collision faults of a rotor system. ${ }^{22}$

Although VMD has been successfully applied to the intelligent fault diagnosis, the parameter optimization and component selection issues remain the most influencing factors for the decomposition performance. In other words, VMD has two important parameters, namely, the number of components $K$ and the penalty factor $a .{ }^{13}$ If these two parameters are not optimized, some errors of signal decomposition will occur and cut down diagnostic accuracy. In addition, it is of great concern to select the sensitive and relevant principal components of VMD from signal decomposition.

To overcome the deficiencies of VMD, a novel optimization algorithm, called particle swarm optimization (PSO), ${ }^{27}$ is employed to optimize the two parameters of VMD in this work. A new well-defined relevant energy $(\mathrm{Re})$ index is also applied to evaluate the reconstructed signals. A designed signal enhancement method (VMD with parameter optimization and components selection index Re) called optimized relevant variational mode decomposition (ORVMD) is presented in this study.

Furthermore, the intelligent recognition algorithm is urgently needed to implement the weak fault detection in an automatic approach. As one of the most advanced data processing and pattern recognition methodologies, deep learning (DL) has been broadly applied in many fields such as machine vision, speech processing and intelligent fault diagnosis. ${ }^{28-35}$ Among them, auto-encoder (AE) ${ }^{34}$ is one of the more widely applied deep learning architectures that can effectively extract the underlying data into high-level and meaningful features. For instance, Sun et al. proposed a novel induction motor fault diagnosis based on a sparse auto-encoder (SAE). ${ }^{35}$ In the work of Junbo et al., a novel intelligent fault diagnosis of rolling bearings built on wavelet transform and AE was developed to enhance the availability of fault diagnosis. ${ }^{36}$

It has been demonstrated that the traditional SAE algorithm is very sensitive to industrial noise. ${ }^{36,37}$ Currently, the sparsity and generalization are beneficial to signal processing and fault diagnosis. ${ }^{37-40}$ As is well-known that $l_{1}$ norm can make the data sparser, and $l_{2}$ norm is employed to prevent over-fitting of data. Therefore, if the advantages of the $l_{1}$ and $l_{2}$ norms are combined, the sparse and generalization performance can be simultaneously improved. ${ }^{37}$ To comprehensively utilize the above-mentioned two types of regularization techniques, a norm combination (termed $l_{1} / l_{2}$ ) is developed to strengthen the generalization of SAE. The so-called improved stacked auto-encoder (ISAE) with the generalized $l_{1} / l_{2}$ norm is constructed in this work.

Specifically, the advantages of the proposed ORVMD algorithm in weak signal processing and the ISAE algorithm in intelligent fault recognition are integrated in this work. A novel intelligent weak fault diagnosis framework based on ORVMD and ISAE algorithms for fault diagnosis of rotating machinery is proposed. The main contributions of this work are outlined as:

1) An optimized VMD algorithm (called ORVMD) is proposed to overcome the deficiencies of the original VMD, which can remove redundant and irrelevant fault components and implement weak signal enhancement.

2) To utilize two types of regularization norms, a new improved stacked Auto-encoder (ISAE) with generalized $l_{1} / l_{2}$ norm is constructed.

3) A new intelligent weak fault recognition framework of rotating machinery built on ORVMD and ISAE is proposed. And this framework is validated with two case studies of vibration signals from roller bearings.

The remainder of this paper is organized as below. A brief review of the background theories is provided in Section 2. In Section 3, two proposed algorithms (ORVMD and ISAE) are presented. In Section 4, the intelligent weak fault diagnosis framework of rotating machinery via ORVMD and ISAE is proposed. In Section 5, the proposed algorithm and fault diagnosis framework are validated with the two cases of experimental signals from roller bearings. Conclusions are given in Section 6.

\section{BACKGROUND}

\subsection{Brief Review of the General VMD}

To obtain an intrinsic mode function (IMF), VMD gets rid of the circular stripping signal used by EMD, and the signal decomposition of VMD is converted into the variational framework. The adaptive decomposition is implemented through an optimal solution search constraint variational model, and then the center frequency and bandwidth are updated..$^{22-24}$ If the input signal $f(t)$ is composed of a series of components with different bandwidths, then the sum of the components is equal to the constraint of the original input signal $x(t)$. Ultimately, the aggregation bandwidth sum of the components is sought to 
be minimum. Its corresponding constraint variational model is rewritten as

$$
\left\{\begin{array}{l}
\min _{\left\{u_{k}\right\},\left\{w_{k}\right\}}\left\{\sum_{k}\left\|\partial_{t}\left[\left(\delta(t)+\frac{j}{\pi t}\right) * u_{k}(t)\right] e^{-j w_{k} t}\right\|_{2}^{2}\right\} ; \\
\text { s.t. } \sum_{k} u_{k}=f ;
\end{array}\right.
$$

where $\left\{u_{k}\right\}$ represents IMF components, $\left\{w_{k}\right\}$ is the frequency center of each component. $\delta(t)$ is the Dirichlet function.

To resolve the above-mentioned constrained variational phenomenon, the Augmented Lagrange (AL) function is introduced as

$$
\begin{aligned}
& L\left(\left\{w_{k}\right\},\left\{u_{k}\right\}\right)= \\
& \alpha \sum_{k}\left\|\partial_{t}\left[\left(\delta(t)+\frac{j}{\pi t}\right) * u_{k}(t)\right] e^{-j w_{k} t}\right\|_{2}^{2}+ \\
& \left\|f(t)-\sum_{k} u_{k}(t)\right\|_{2}^{2}+\left\langle\lambda(t), f(t)-\sum_{k} u_{k}(t)\right\rangle ;
\end{aligned}
$$

where $\alpha$ is the penalty parameter, and $\lambda$ is the Lagrange multiplier. Subsequently, the so-called Alternate Direction Method of Multipliers (ADMM) is employed to obtain the "saddle point". By performing a loop, $u_{k}$ and $w_{k}$ are timely updated. For the sake of finding the optimal solution of the constrained variational model in Eq. (2), the original signals are decomposed into $K$ narrow-band components. Thus, the value of $u_{k}$ is described as

$$
\begin{aligned}
& u_{k}^{n+1}= \\
& \arg \min \left\{\alpha \sum_{k}\left\|\partial_{t}\left[\left(\delta(t)+\frac{j}{\pi t}\right) * u_{k}(t)\right] e^{-j w_{k} t}\right\|_{2}^{2}+\right. \\
& \left.\left\|f(t)-\sum_{k} u_{k}(t)+\frac{\lambda(t)}{2}\right\|_{2}^{2}\right\}
\end{aligned}
$$

where Eq. (3) is transformed into the frequency domain, the updated frequency domain of each mode is obtained as

$$
\hat{u}_{k}^{n+1}(w)=\frac{\hat{f}(w)-\sum_{i \neq k} \hat{u}(w)+\frac{\hat{\lambda}(w)}{2}}{1+2 \alpha\left(w-w_{k}\right)^{2}} .
$$

According to the same calculation process, the central frequency is converted into the frequency domain. Its central frequency is updated by

$$
w_{k}^{n+1}=\frac{\int_{0}^{\infty} w\left|\hat{u}_{k}(w)\right|^{2} d w}{\int_{0}^{\infty}\left|\hat{u}_{k}(w)\right|^{2} d w}
$$

where $u_{k}^{n+1}$ is equivalent to the Wiener Filter for the current residual amount $\hat{f}(w)-\sum_{i \neq k} \hat{u}(w)$ where $w_{k}$ is the power spectrum center of the current mode function. $\left\{u_{k}\right\}$ is calculated using an inverse Fourier transform.

\subsection{Brief Review of the General SAE}

Traditional signal analysis has difficultly accurately diagnosing a fault when the fault impulse signal is not obvious.
Thus, an intelligent diagnosis based on DL is needed to implement fault recognition. SAE is composed of several AEs. Where the mapping function from inputting layer to encoding layer is usually a nonlinear function, which is described as

$$
h_{i}=f\left(x_{i}\right)=\frac{1}{1+\exp \left(-\left(w x_{i}+b\right)\right)} ;
$$

where $w$ is the weight between layers and $b$ is the deviation. Similarly, the nonlinear function from the encoding layer to the decoding layer can be written as

$$
x_{i}^{\prime}=g\left(h_{i}\right)=\frac{1}{1+\exp \left(-\left(w^{\prime} h_{i}+b^{\prime}\right)\right)} ;
$$

where $w^{\prime}$ is weight, and $b^{\prime}$ is the deviation. The objective optimization is described as

$$
J=L\left\{x_{i}^{\prime}, x\right\}=\min \frac{1}{N} \sum_{i=1}^{N}\left\|x_{i}^{\prime}-x_{i}\right\| .
$$

\section{THE PROPOSED ALGORITHMS: ORVMD AND ISAE}

\subsection{Improved Signal Enhancement Method: ORVMD}

Compared with the general VMD algorithm, the core of the proposed ORVMD can take advantage of parameter optimization and the new well-defined index to select sensitive principal components. The specific step of the ORVMD method are presented in the remainder of this section.

\subsubsection{Parameter Optimization and Signal Decomposition}

The penalty coefficient $a$ and the number of decomposition $k$ in the VMD algorithm have a great influence on signal decomposition and reconstruction. Owing that the signal decomposition and reconstruction are changeable, the parameter combination $[k, a]$ is difficult to be determined in advance. Particle swarm optimization (PSO) ${ }^{27,41}$ has a desired global optimization ability. To avoid the interference of human subjective factors, the PSO algorithm is used to enhance the parameter optimization capability of the VMD method in this work.

For $D$ dimensional space, the population of $N$ particles is $X X=\left(X X_{1}, X X_{2}, \ldots, X X_{N}\right)$, the position of the $i$ th particle in the $d$-dimensional search space is $X X_{i}=$ $\left(x_{i 1}, x_{i 2}, \ldots, x_{i D}\right)$. The velocity of the $i$-th particle is $V_{i}=\left(v_{i 1}, v_{i 2}, \ldots, v_{i D}\right)$, the individual local extreme value is $P_{i}=\left(p_{i 1}, p_{i 2}, \ldots, p_{i D}\right)$, the global extreme value is $G=$ $\left(g_{1}, g_{2}, \ldots, g_{D}\right)$, each particle can update its speed and position through local extremum and population global extremum, as described by

$$
\left\{\begin{array}{l}
v_{i d}^{k+1}=w v_{i d}^{k}+c_{1} \xi\left(g_{d}^{k}-x_{i d}^{k}\right) \\
x_{i d}^{k+1}=v_{i d}^{k+1}+x_{i d}^{k}
\end{array}\right.
$$

where $w$ is the inertia weight, $k$ is the current iteration number. In the publication by Yan et al.., ${ }^{13}$ the envelope signals obtained after signal demodulation are processed into a probability distribution sequence, and the envelope entropy value 


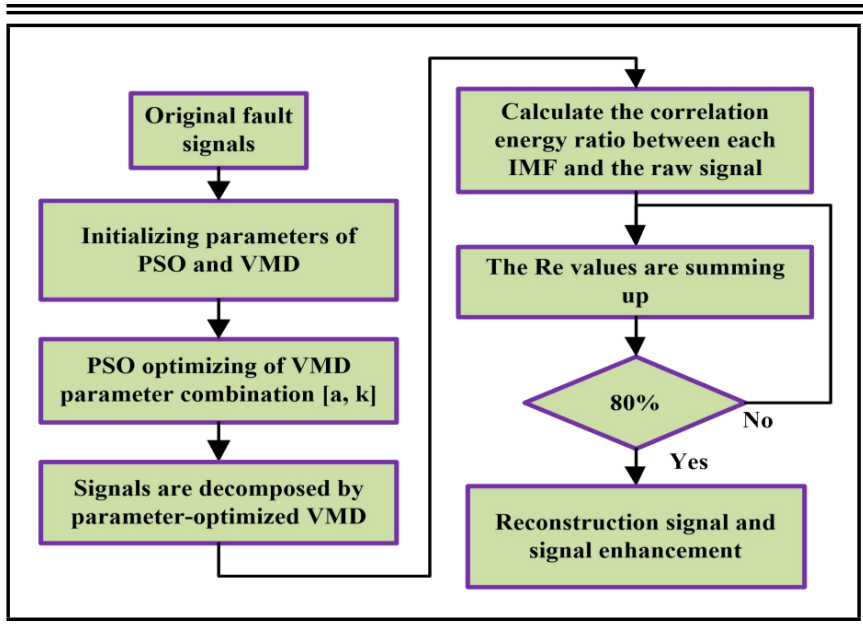

Figure 1. The illustration of the proposed signal enhancement algorithm (ORVMD).

(EEV) is calculated according to the sparse characteristics of the original signals. After the fault signals are decomposed by the parameter optimized VMD, the sparsity of the component signals is weak, its envelope entropy value (EEV) is larger. Conversely, the envelope entropy (EEV) is smaller. As a result, the local minimum envelope entropy is deemed as the fitness value of the optimized VMD in this study.

\subsubsection{Component Selection and Signal Reconstruction}

To remove redundancy components, the defined component selection index combines the concept of relevance coefficient and energy ratio. The relevant coefficient $R\left(x_{i}, x_{j}\right)$ is applied to describe the related degree between the statistical vector $x_{i}$ and $x_{j}{ }^{42}$ Therefore, relevant coefficient is defined as

$$
R\left(x_{i}, x_{j}\right)=\frac{E X\left(x_{i}-E X\left(x_{i}\right)\right)-E X\left(x_{j}-E X\left(x_{j}\right)\right)}{\sqrt{D\left(x_{i}\right)} \sqrt{D\left(x_{j}\right)}} ;
$$

where $E X\left(x_{i}\right)$ and $E X\left(x_{j}\right)$ are mathematical expectation, $D\left(x_{i}\right)$ and $D\left(x_{j}\right)$ are variance. To be specific, the proposed $R e$ index is defined as

$$
\operatorname{Re}(i m f(i))=\frac{E(i m f(i)) * R(x, i m f(i))}{E_{\text {total }}} ;
$$

where $E(\operatorname{imf}(i))$ is the energy of component signals, $E_{\text {total }}$ is the energy of the raw signals. Consequently, as the value of $R e$ is increased, the correlation with the fault signal component is increased. Compared with the single relevance coefficient or energy ratio, the proposed $R e$ index can efficiently reflect fault information. When the cumulative component index reaches more than $80 \%$, signal reconstruction can be carried out.

In summary, the steps of the ORVMD algorithm are:

- Initialize the parameters of the PSO and the fitness function. The parameter combination $[a, k]$ is regarded as a particle position, a certain parameter combination as the initial particle position can be randomly generated, its moving speed of each particle was initialized.

- The original signals are decomposed via VMD at different particle positions to calculate the fitness value of each particle position.

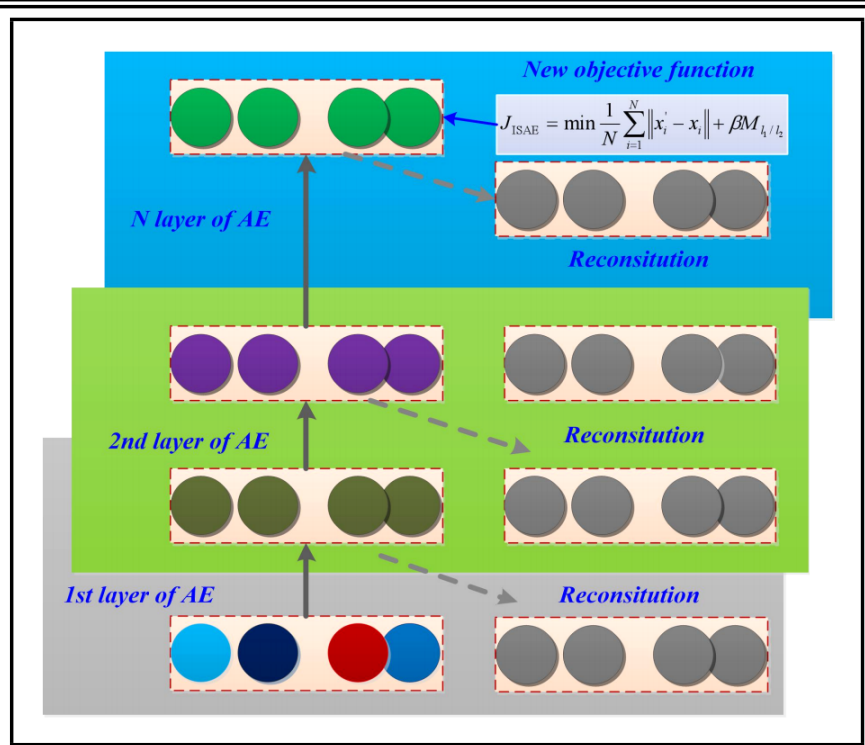

Figure 2. The structure illustration of the proposed ISAE algorithm.

- Comparing with the different fitness values, the individual local extreme value, the population global extreme value, the velocity and position of particles are updated.

- Loop iteration, go to step 3 until the iteration number reaches the maximum setting value.

- After optimized combination parameters, the $R e$ value of IMF components was calculated and sorted it from large too small. When the cumulative $R e$ value reached to $80 \%$, the filtered signal components are reconstructed to obtain the enhanced signals.

The proposed ORVMD algorithm is illustrated in Fig. 1.

\subsection{Intelligent Fault Recognition Algorithm: Improved Stacked Auto-Encoder (ISAE)}

It is illustrated that the SAE algorithm is not robust. ${ }^{34-36}$ Fortunately, the sparsity and generalization are the increasingly important research topic in recent years. The generalized $l_{p}$ norm and its combined norm are a large family of sparse measures. ${ }^{34-37}$ The $l_{p}$ norm can enhance the sparseness and generalized performance of data. The $l_{1}$ is a well-known norm, which can make the data sparser, and the $l_{2}$ norm can prevent over-fitting of data. To utilize the above-mentioned regularization techniques, the norm combination $\left(l_{1} / l_{2}\right)$, i.e. combining with $l_{1}$ and $l_{2}$ norms is developed to up-grade the generalization of the general SAE algorithm. This is termed as an improved stacked auto-encoder (ISAE). Therefore, the $l_{1}$ norm is expressed as the sum of absolute values of each element of $\mathrm{w}$ vector, which can be written as

$$
l_{1}=\|w\|_{1}=\sum_{i=1}^{n}\left|w_{i}\right|
$$

The $l_{1}$ norm is usually sparse to choose eigenvectors with a small number. The $l_{2}$ norm is the $1 / 2$ power of the sum of squares for each element of $\mathrm{w}$ vector. Accordingly, $l_{2}$ norm is called as Euclidean norm (Euclidean distance)

$$
l_{2}=\|w\|_{2}=\sqrt{\sum_{i=1}^{n} w_{i}^{2}} .
$$




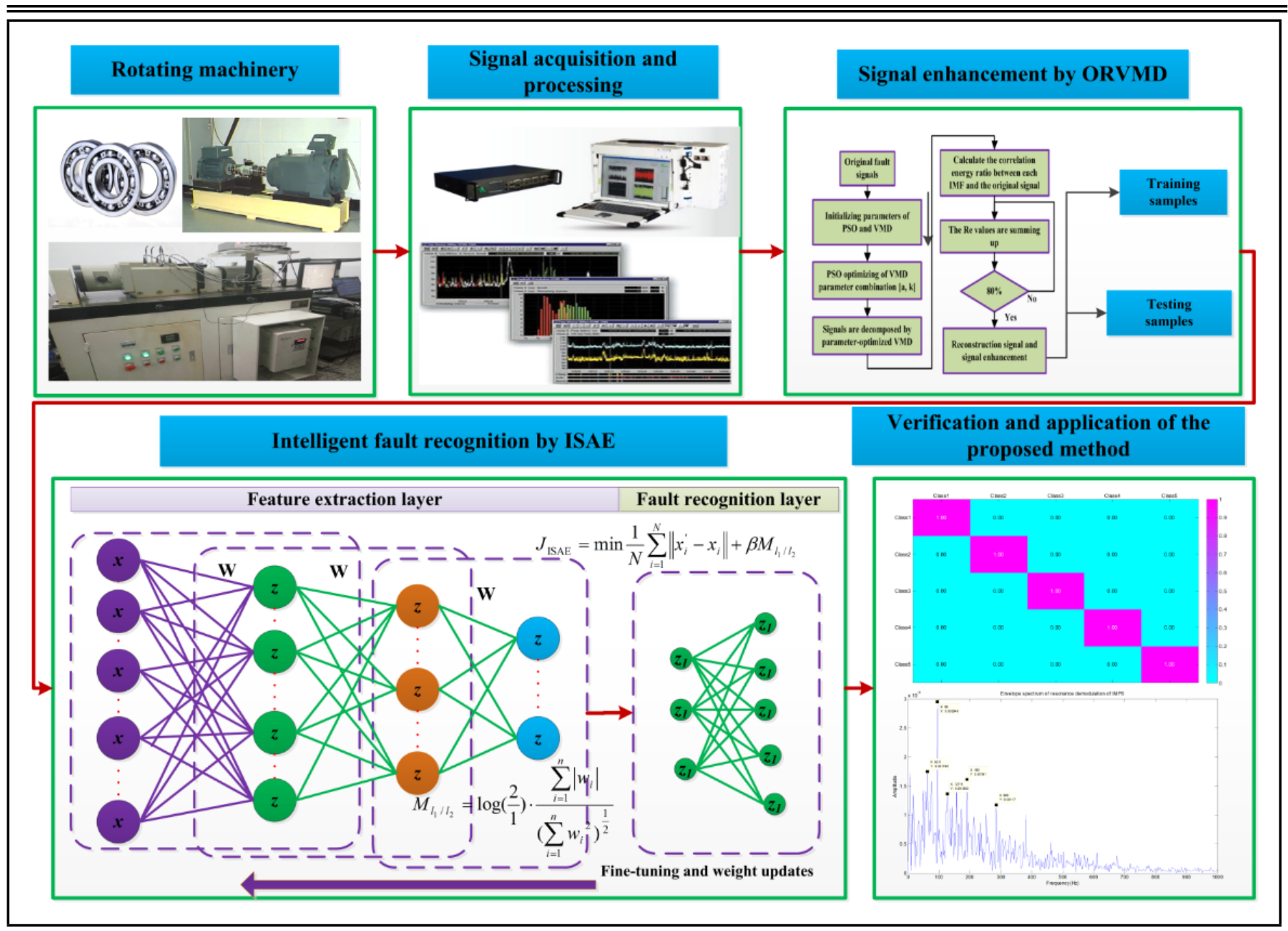

Figure 3. The flowchart of the proposed intelligent weak fault recognition for rotating machinery.

According to Vincent et al., ${ }^{34}$ the $l_{1} / l_{2}$ norm can be obtained as

$$
l_{1} / l_{2}=\frac{\|w\|_{1}}{\|w\|_{2}}=\frac{\sum_{i=1}^{n}\left|w_{i}\right|}{\left(\sum_{i=1}^{n} w_{i}^{2}\right)^{1 / 2}} .
$$

Moreover, according to the proposition by Jia et al., ${ }^{37} l_{1} / l_{2}$ norm can be normalized into a general framework, which is given as

$$
M_{l_{1} / l_{2}}=\log \left(\frac{2}{1}\right) \cdot \frac{\sum_{i=1}^{n}\left|w_{i}\right|}{\left(\sum_{i=1}^{n} w_{i}^{2}\right)^{1 / 2}} .
$$

From the perspective of Bayesian learning, the regularization term of combination norm has such a function that if the neural unit of the neural network relates to the unit, it is prominent, otherwise, it is 0 . Therefore, a combination norm is pushed under the condition of prior knowledge, which can make the model's weight generalization ability stronger. To sum up, the new objective function of ISAE is defined as

$$
J_{\mathrm{ISAE}}=\min \frac{1}{N} \sum_{i=1}^{N}\left\|x_{i}^{\prime}-x_{i}\right\|+\beta M_{l_{1} / l_{2}} ;
$$

where $\beta$ is the norm adjustment parameter. In the study by Zhao et al. ${ }^{33}$ and Sun et al..$^{35}$ the training process of the ISAE is the same as the RBM by using the greedy layer-by-layer pertraining. The ISAE (as described in Fig. 2) is a deep neural network composed of multiple-layer improved auto-encoders.

\section{THE PROPOSED INTELLIGENT WEAK FAULT RECOGNITION FRAMEWORK}

Combining the advantages of the ORVMD and the ISAE, an intelligent weak fault recognition framework is proposed and divided into two stages:

Stage1: Weak signal enhancement stage

Step1: Several sensors are applied to capture the vibration signals from rotating machinery.

Step2: Initializing combination parameter and the fitness function, the optimized parameter combination is obtained.

Step3: The raw signals are decomposed and reconstructed via the ORVMD.

Step4: The enhanced signals are converted into spectral signals, which are divided into training samples and testing samples, respectively.

Stage2: Intelligent recognition stage

Step5: Initializing the parameters of the ISAE.

Step6: Using training samples to train the ISAE. 


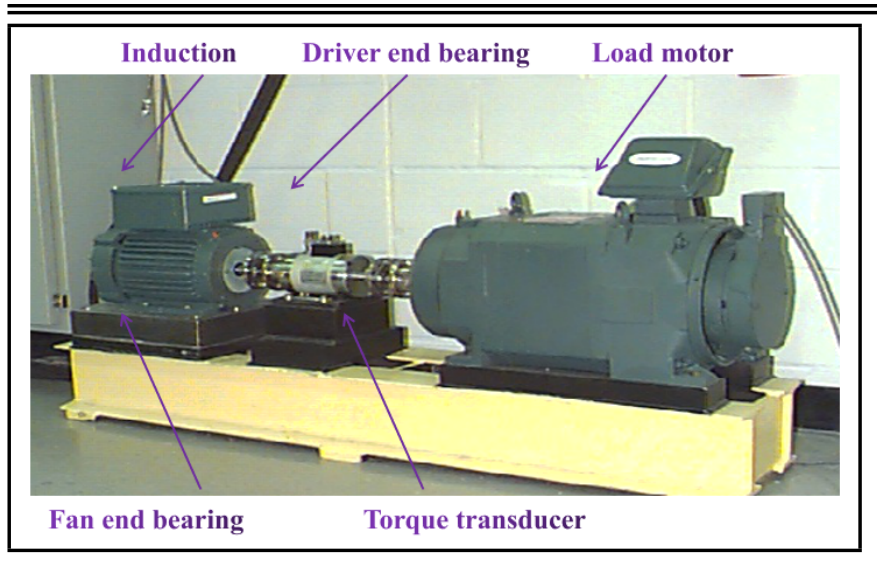

Figure 4. The rolling bearing test rig of Case Western Reserve University (CWRU).

Table 1. The established rolling bearing data set under different health condition of CWRU.

\begin{tabular}{||c|c|c|c||}
\hline Fault types & $\begin{array}{c}\text { Depth of } \\
\text { fault (mm) }\end{array}$ & $\begin{array}{c}\text { Fault } \\
\text { label }\end{array}$ & $\begin{array}{c}\text { Number of } \\
\text { samples }\end{array}$ \\
\hline Rolling element slight fault & 0.18 & F1 & 100 \\
Rolling element serious fault & 0.54 & F2 & 100 \\
Inner ring slight fault & 0.18 & F3 & 100 \\
Inner ring serious fault & 0.54 & F4 & 100 \\
Outer ring slight fault & 0.18 & F5 & 100 \\
Outer ring serious fault & 0.54 & F6 & 100 \\
Normal condition & 0 & F6 & 100 \\
\hline
\end{tabular}

Step7: Fine tuning the model parameters and saving the trained network parameters.

Step8: Test samples are fed into the trained ISAE for fault diagnosis.

Furthermore, the flowchart of fault recognition framework is described in Fig. 3.

\section{EXPERIMENTAL VALIDATION AND ANALYSIS}

In this section, the availability of the proposed fault recognition framework is validated with two diagnostic cases of rolling bearing data set from the electrical engineering laboratory (Case Western Reserve University (CWRU) $)^{43}$ and the full-life testing bench of Southeast University (Accelerated Bearing Life Tester (ABLT-1A)), respectively.

\subsection{Experimental Analysis with Rolling Bearing Weak Failure of CWRU}

Generally, rolling bearing fault signals have strong nonstationary characteristics in practical industrial engineering. Thus, their fault characteristic information is weak. Consequently, the development and research of intelligent fault diagnosis technology have a positive practical significance.

For further validate the availability of the proposed framework in fault recognition, the experimental data used in this section originated from the rolling bearings experimental data of Case Western Reserve University (CWRU) in the United States. Specifically, the selective rolling bearing was a 6205JEM SKF deep groove ball bearing. It is well-known that its internal diameter, external diameter, and thickness rolling element diameter are $25 \mathrm{~mm}, 52 \mathrm{~mm}, 15 \mathrm{~mm}$ and $8.18 \mathrm{~mm}$, respectively. Subsequently, vibration signals of rolling bearing were collected at a load of $1 \mathrm{hp}$, a rotational speed of
Table 2. The parameter setting of the proposed ORVMD and ISAE algorithms.

\begin{tabular}{||c|c|}
\hline Parameter name & Value \\
\hline Maximum evolutionary algebra (ORVMD) & $G_{\max }=10$ \\
Population size $M$ (ORVMD) & $M=10$ \\
Maximum number of chaotic search (ORVMD) & $\operatorname{ax}_{k}=10$ \\
Acceleration constant (ORVMD) & $c_{1}=2$ \\
Acceleration constant (ORVMD) & $c_{2}=2$ \\
Optimization objective (ORVMD) & Envelope entropy \\
Local minimum envelope entropy (ORVMD) & Sixth generation \\
Best parameter (ORVMD) & $a=830$ \\
Best parameter (ORVMD) & $k=7$ \\
Nodes (ISAE) & $1024-400-100-6$ \\
Lumber of iterations (ISAE) & 200 \\
Batch size (ISAE) & 20 \\
Regularization (ISAE) & 0.1 \\
Sparsity Target (ISAE) & 0.05 \\
Weight Penalty (ISAE) & 0 \\
Norm parameter (ISAE) & 0.5 \\
Activation function (ISAE) & tanh \\
\hline
\end{tabular}

Table 3. The index for IMF component signals.

\begin{tabular}{|c|c|c|}
\hline Component signals & Relevant energy $(R e)$ & Relevant coefficient $(R c)$ \\
\hline IMF1 & 0.0146 & 0.2522 \\
IMF2 & 0.0512 & 0.3855 \\
IMF3 & 0.0674 & 0.4471 \\
IMF4 & 0.1182 & 0.5122 \\
IMF5 & 0.0424 & 0.3750 \\
IMF6 & 0.1059 & 0.4942 \\
IMF7 & 0.0391 & 0.3912 \\
\hline
\end{tabular}

$1750 \mathrm{r} / \mathrm{min}$, and a sampling frequency of $12 \mathrm{kHz}$. The failure of rolling bearing in this section was manufactured and its testbed is described in Fig. 4. Moreover, 7 kinds of rolling bearing health conditions were studied in this case study. They were the outer ring slight fault; outer ring serious fault; inner ring slight fault; inner ring serious fault; rolling element slight fault; rolling element serious fault and normal condition. Accordingly, the statistical summary of the data set is illustrated in Table 1. Among them, 50 groups were randomly taken as training samples for each health condition, while the remaining 50 groups were taken as testing samples.

To reveal different degrees of failure, the waveform of different kind of vibration signals in the time domain and unilateral spectrum frequency domain are drawn in Fig. 5. Weak fault signals are significantly affected by noise and irrelevant interference, and their impact characteristics are not obvious. Also, the resonance band in the spectrum was not obvious. Thus, weak faults were difficult to be observed.

According to Fig. 3, the proposed method can be roughly divided into two stages: weak signal enhancement and intelligent recognition. To decompose and reconstruct the weak signals and other signals, the proposed ORVMD was applied to reconstruct the raw signals. Limited by the length of this study, the vibration signals of inner ring weak failure were regarded as an example to decompose and enhance signals by using the ORVMD algorithm.

\subsubsection{Signal Enhancement by the Proposed ORVMD}

To be specific, ORVMD algorithm can be devoted to decomposed and reconstruct the weak failure signals of rolling bearing inner ring. According to Yan et al. ${ }^{13}$ and Chenglin et al., ${ }^{41}$ the parameters of particle swarm optimization algorithm were set up as Table 2. The local minimum envelope entropy in the process of particle swarm optimization with the 
Time domain waveform of weak fault signal of bearing rolling body

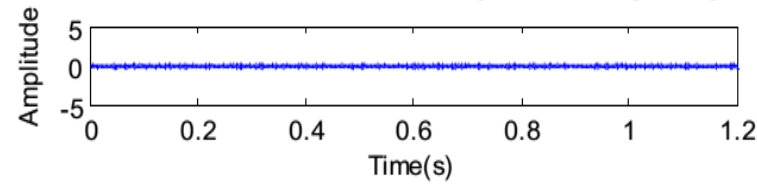

Time domain waveform of serious fault signal of bearing rolling body

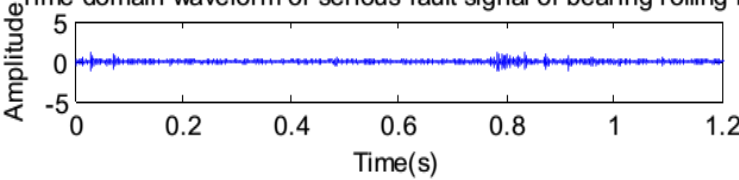

Time domain waveform of weak fault signal of bearing inner ring

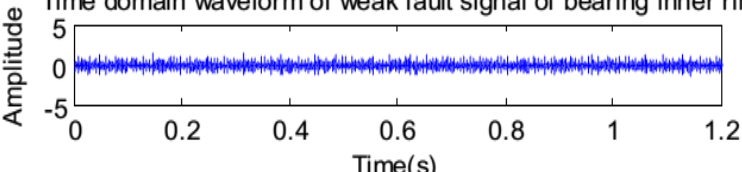

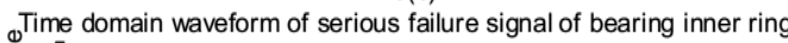

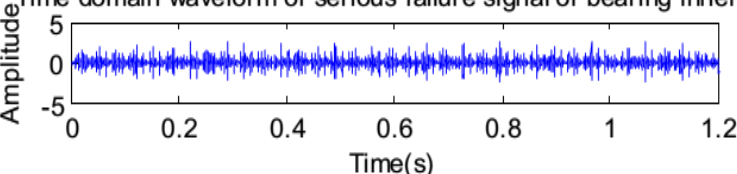

Time domain waveform of weak fault signal of bearing outer ring
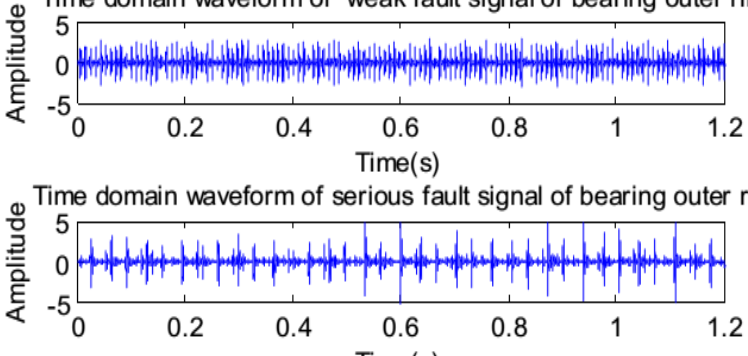

Frequency domain waveform of weak fault signal of bearing rolling body

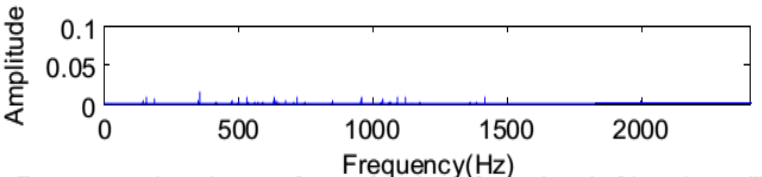

Frequency domain waveform of serious fault signal of bearing rolling body

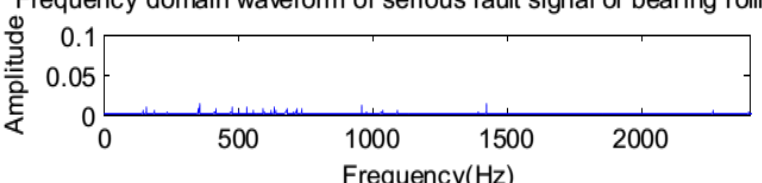

Frequency domain waveform of weak fault signal of bearing inner ring

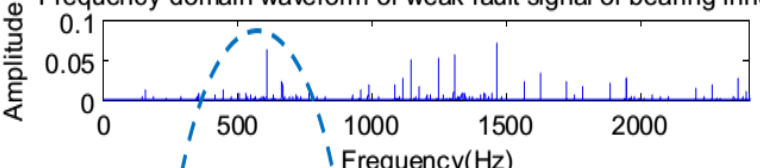

Frequency dqmain waveform of serious failure signal of bearing inner ring

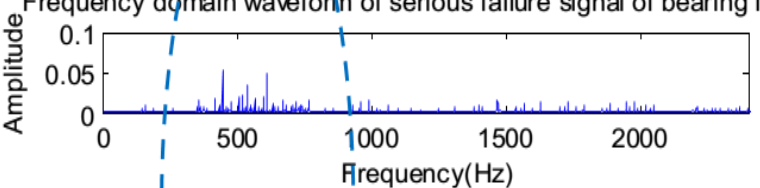

. Frequency domain waveform of weak fault signal of bearing outer ring

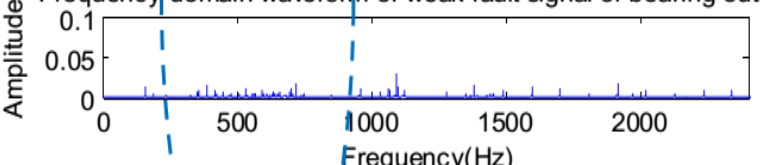

Time domain waveform off serious fault signal of bearing outer ring

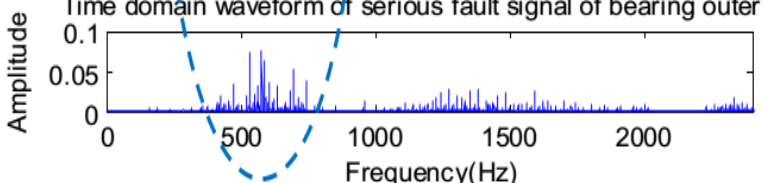

Figure 5. The waveform of vibration signals in the time domain and frequency domain.

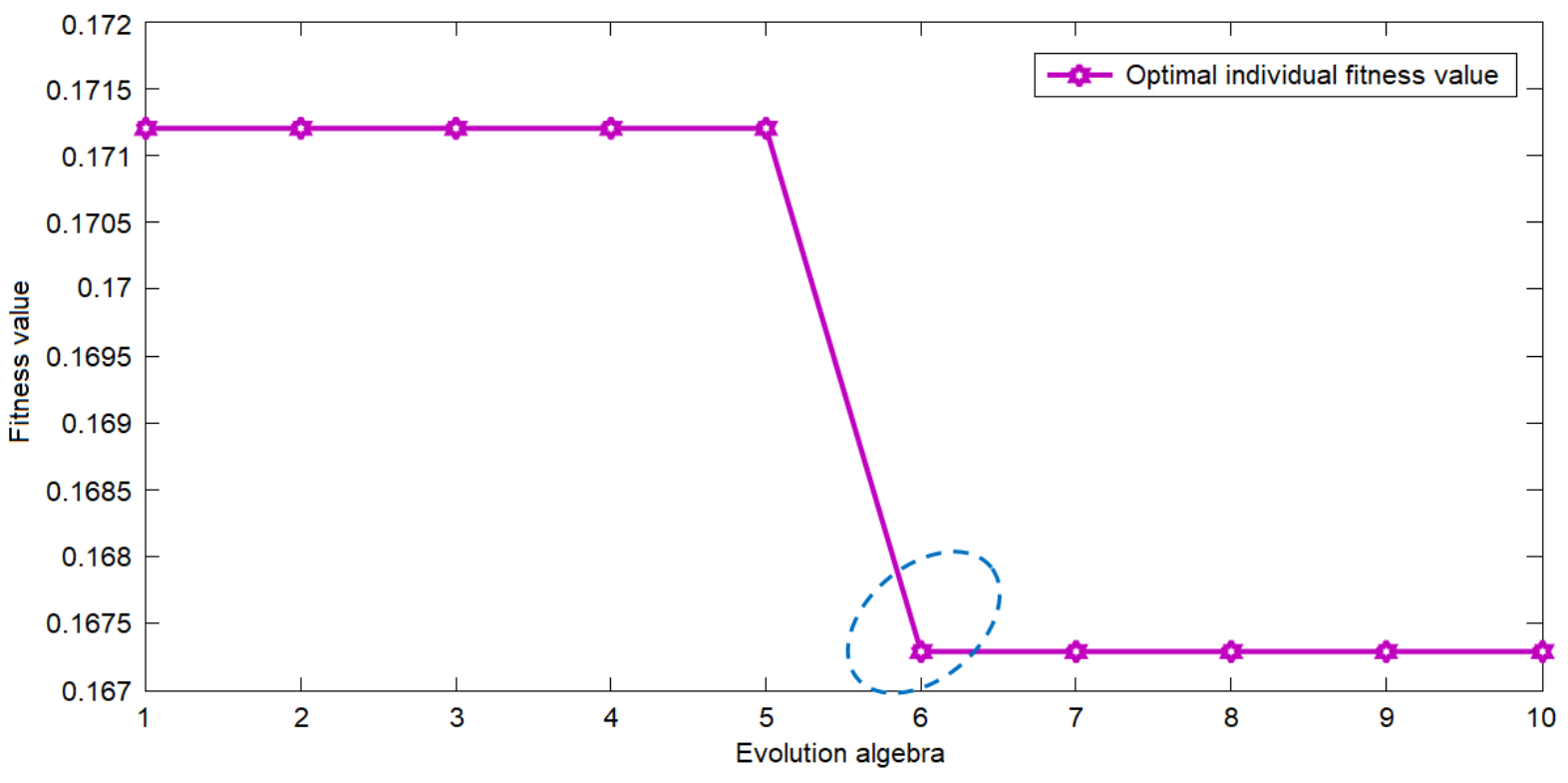

Figure 6. The minimum envelope entropy of inner ring weak fault signal (CWRU) via ORVMD.

evolution algebra is decomposed in Fig. 6. Apparently, the local minimum envelope entropy value was in the sixth generation. Thus, the best parameter combination can be selected as $[a, k]=[830,7]$. Naturally, its inner ring weak failure vibration signals was decomposed and reconstructed via the ORVMD.
Specifically, component signals via the ORVMD are drawn in Fig. 7. Compared with other component signals, the regular impact in the waveform of the IMF4 was more obvious, and its frequency band center was just at $3000 \mathrm{~Hz}$. For the vicinity of the resonance frequency, the envelope entropy of the IMF4 for all the components was also the smallest. Fur- 


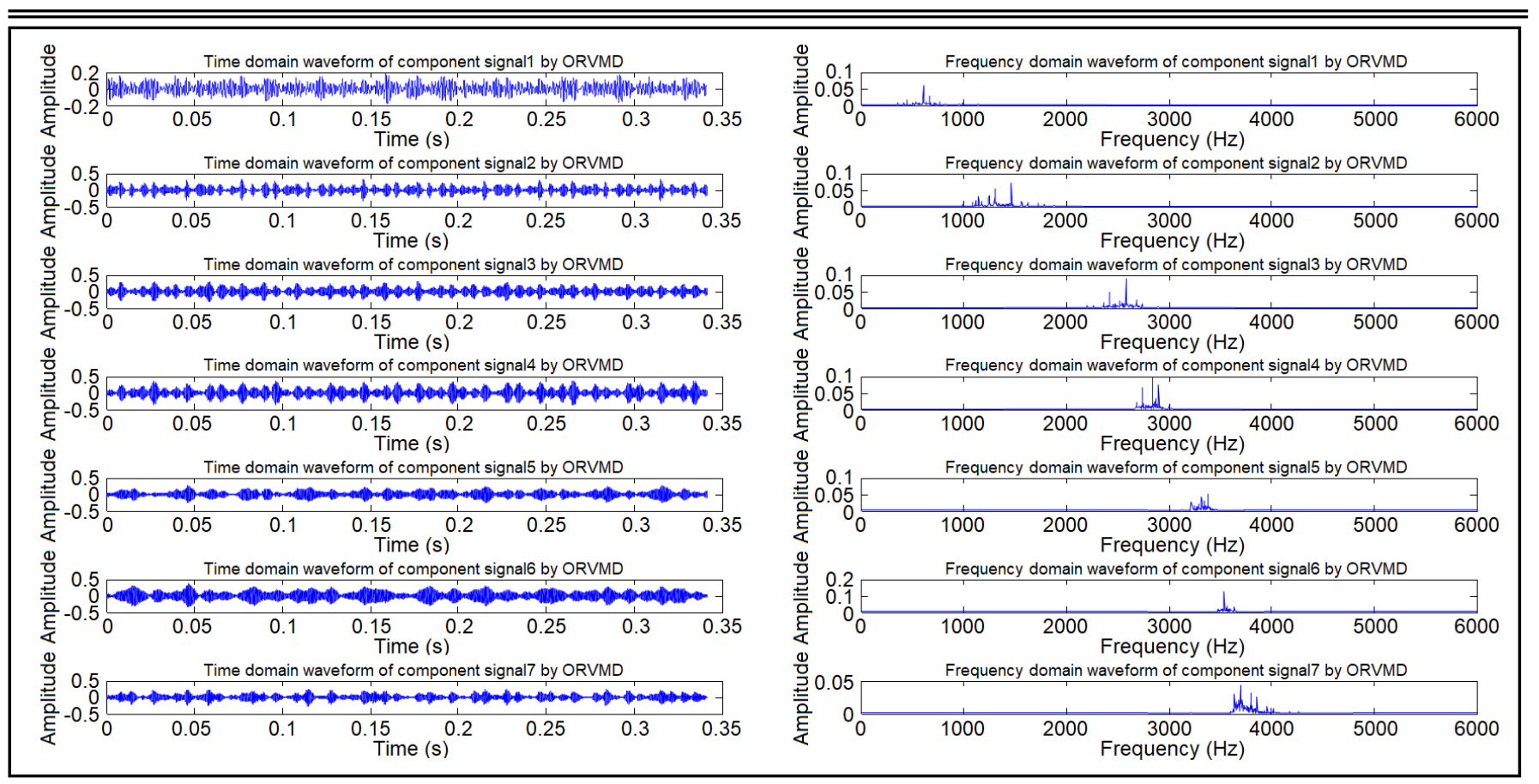

Figure 7. Time and frequency domain waveform of the decomposed inner ring weak fault components.

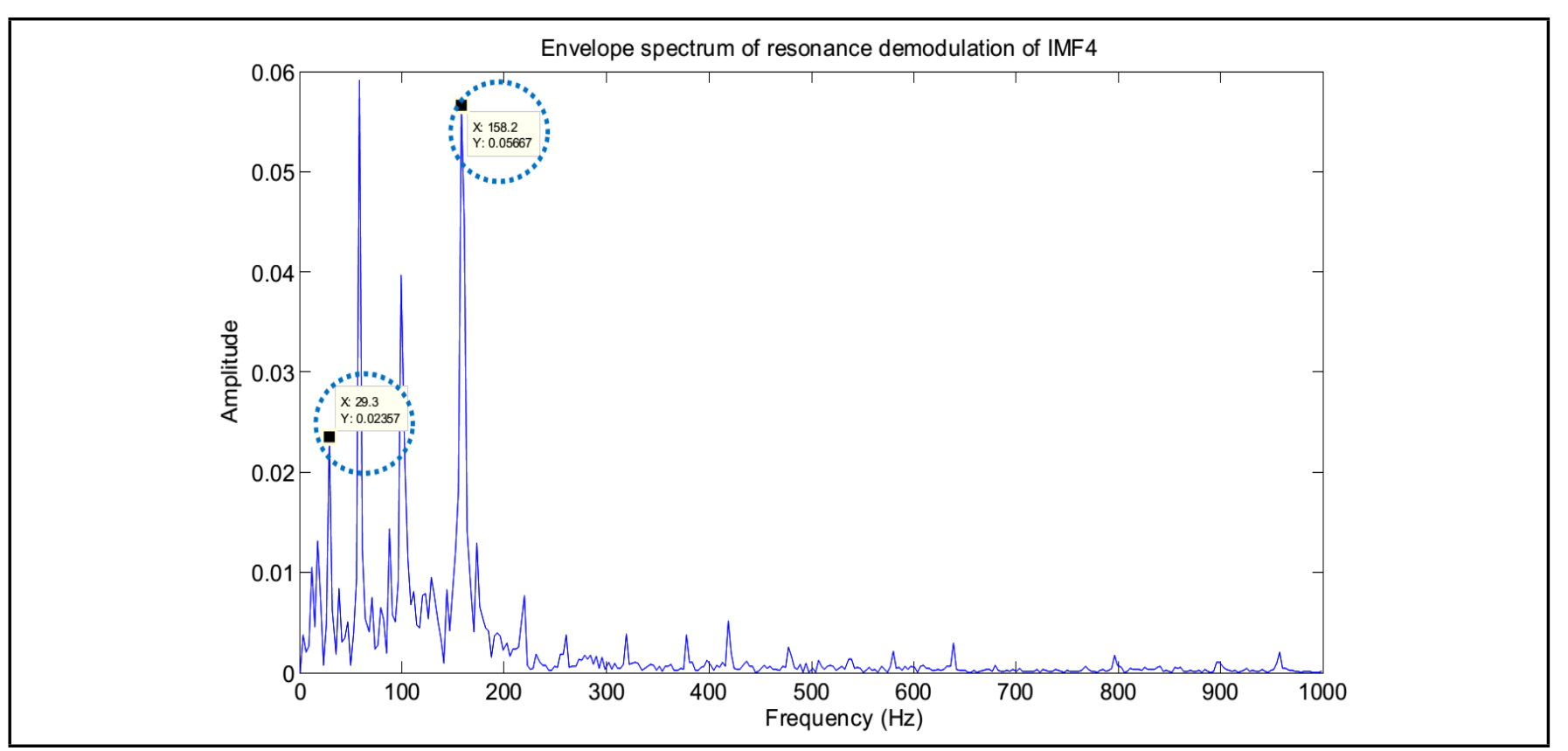

Figure 8. The envelope spectrum of the decomposed inner ring weak fault component (IMF4).

thermore, the envelope spectrum via envelope demodulation is described in Fig. 8 and Fig. 9, in which the line amplitude of the spectrum at the frequency multiplier and the characteristic frequency were prominent. The results demonstrated that the characteristic frequency features were accurately extracted.

The ORVMD with parameter optimization can decompose fault signals from high frequency to low frequency. Hence, its fault vibration signal feature information was expanded. Afterwards, the weak signal was reconstructed by the new components screened index (relevant energy, Re). According to the concept of $R e$ index, the $R e$ index and relevant coefficient $(R c)$ between 7 components (IMF) and the original signal was calculated in Table 3.

The comparison with the original relevant coefficient and energy index is illustrated in Table 2. The new index $(R e)$ highlighted the correlation degree between different components and the original signal. The cumulative Relevant Energy index was equal to 0.8 , which fully covered the main information for signal reconstruction. The reconstructed signal is displayed in Fig. 9. The envelope spectrum showed that its fault frequency is more prominent, which is observed in the figure. The reconstructed signals can be directly observed at the frequency component of $158.2 \mathrm{~Hz}$ at the generation. However, the above-mentioned signal processing methods are in the signal enhancement stage, but the enhanced signal processing methods still needed an effective intelligent recognition algorithm. In other words, the traditional vibration signal analysis based on time domain and frequency domain cannot quantify the characteristics of the mechanical fault information. Therefore, an effective fault recognition framework was required. 


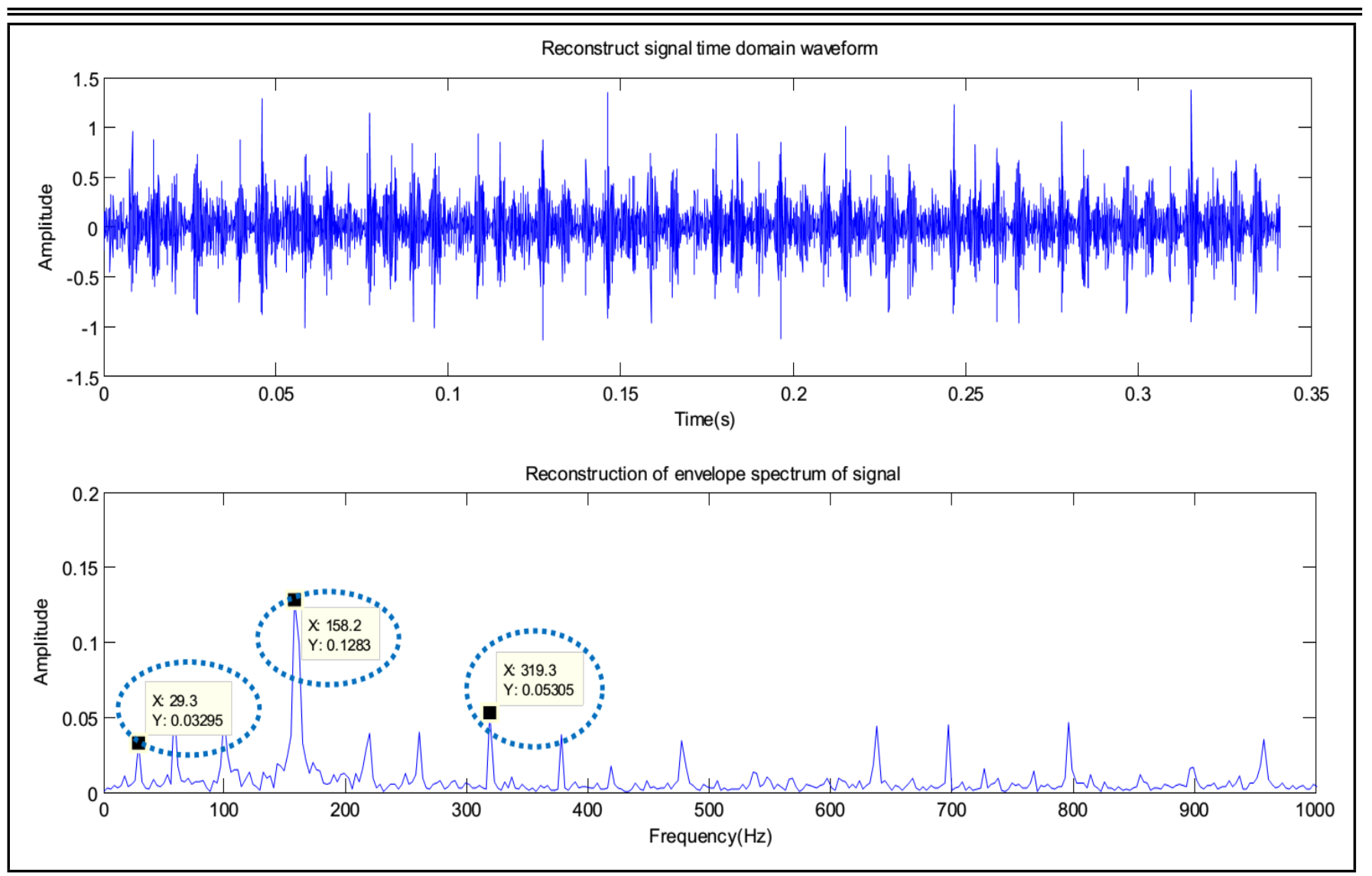

Figure 9. Time-frequency domain waveform and envelope spectrum of the reconstructed inner ring weak fault signal.

\subsubsection{Intelligent Fault Recognition by the Proposed ISAE}

According to Zhao et al., ${ }^{44}$ ISAE was set as two-layer AE, and the number of nerve units was 1024-400-100-6. To sum up, the detailed parameters of ISAE are described in Table 2. For an experimental comparison, three kinds of signal processing method, namely, the original signal, signal processed by VMD and signal processed by ORVMD (regard as OS-ISAE, VMD-ISAE, and ORVMD-ISAE, respectively.) were selected for comparison,. Thus, the fault recognition results of the three methods are obtained in Fig. 10.

Compared with other frameworks, the proposed framework can achieve higher diagnostic accuracy.

\subsubsection{Feature Visualization via t-SNE}

To visualize the above-mentioned diagnosis results, the last layer of extracted features was visualized by using t-SNE (tdistributed stochastic neighbor embedding). ${ }^{45}$ At present, $\mathrm{t}$ SNE technology is widely used in data visualization, to understand the diagnosis model \{original signal + ISAE = FD1; $\mathrm{VMD}+\mathrm{ISAE}=\mathrm{FD} 2$; ORVMD + ISAE $=$ FD3 $\}$. T-SNE is applied to convert high dimensional representation into a twodimensional scatter plot. The above-mentioned three diagnostic methods can obtain the two-dimensional scatter distribution diagram of characteristic points through t-SNE as described in Fig. 11.

As illustrated in Fig. 11, only the ORVMD-ISAE baseddiagnostic model was able to divide the different samples of various conditions, which indicates the effectiveness of the proposed framework.

\subsection{Experimental Analysis with Rolling Bearing Weak Failure of ABLT-1A}

\subsubsection{Data Collection and Parameter Setting}

In this section, the rolling bearing data collected via ABLT1A were applied to validate the effectiveness of the proposed diagnosis framework. In view of this point, the wire cutting was employed to simulate different rolling bearing faults in this section, and the damaged bearings were installed on the accelerated bearing life tester (ABLT-1A provided by Hangzhou Bearing Test Research Center, its structure diagram is shown in the Fig. 12). Also, the corresponding vibration signals were collected under different health conditions. Correspondingly, the failure condition of rolling bearing is drawn in the Fig. 13.

The combination of the measuring instrument with data acquisition card and PCB acceleration sensor was used to pick up the five kinds of rolling bearing fault vibration signals under different fault conditions (i.e. inner ring fault, inner-outer ring compound fault, inner-outer ring weak compound fault, outer ring-ball compound fault and outer ring-ball weak compound fault, which were marked as F1, F2, F3, F4, and F5, respectively) were used for signal acquisition. More precisely, its specific parameters of the experiment are represented in Table 4 . At the same time, to facilitate the subsequent work, every 1024 points of each type of fault signals were intercepted as one fault sample, and 100 samples were obtained for each fault condition. 50 groups were randomly used as training samples, and the remaining groups are used as testing samples. 
(a)

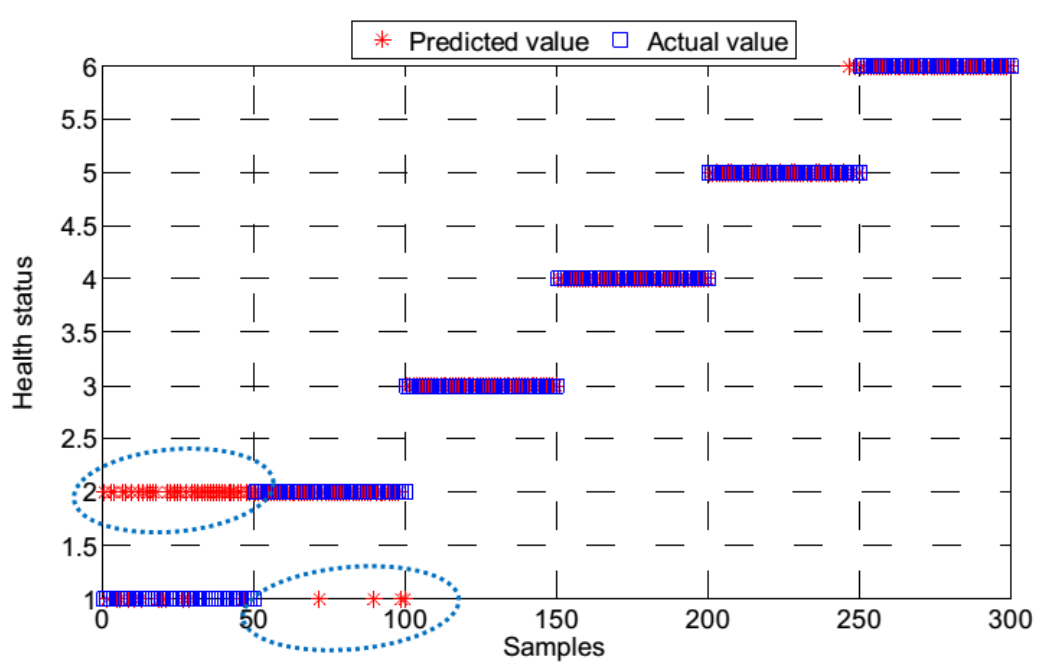

(b)
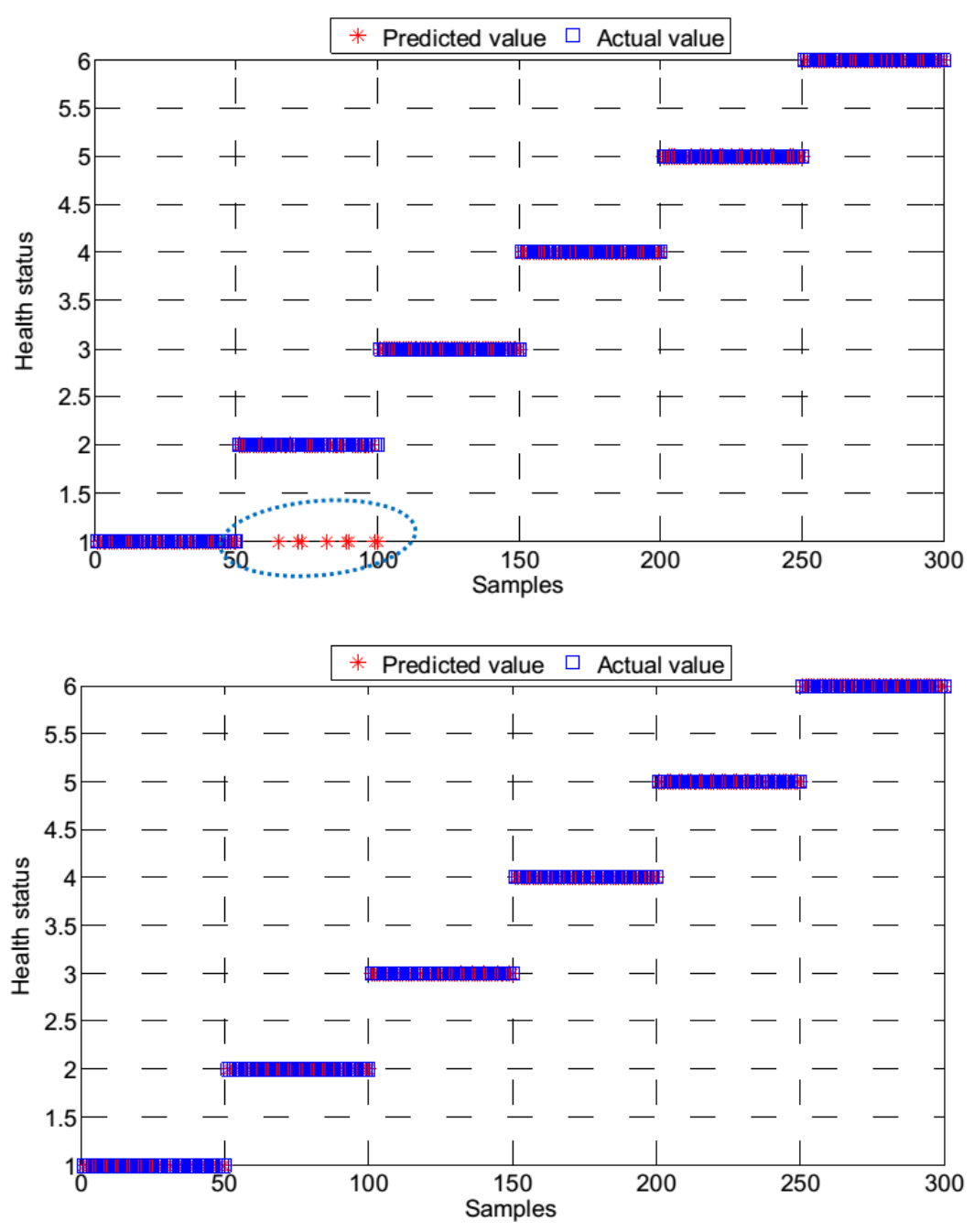

Figure 10. Classification results: a) OS-ISAE; b) VMD-ISAE; c) ORVMD-ISA.

\subsubsection{Signal Processing Results of Experimental Data}

The waveform of the time domain and unilateral spectrum frequency domain are drawn in Fig. 14. Weak fault signals were greatly affected by noise and irrelevant interference, and their impact characteristics were not obvious. Also, the resonance band in the spectrum was not obvious. Thus, weak faults were difficult to be detected.
Moreover, Fig. 15 analyzed the simulation signal by using the proposed framework. Namely, the local minimum envelope entropy value located in the 7-th generation, the best parameter combination is $[a, k]=[905,8]$.

The waveform of different components signals via ORVMD are described in Fig. 16. Compared with other components, the regular impact in the waveform of IMF6 was more obvious. The frequency band center was located at $3000 \mathrm{~Hz}$. Near the 


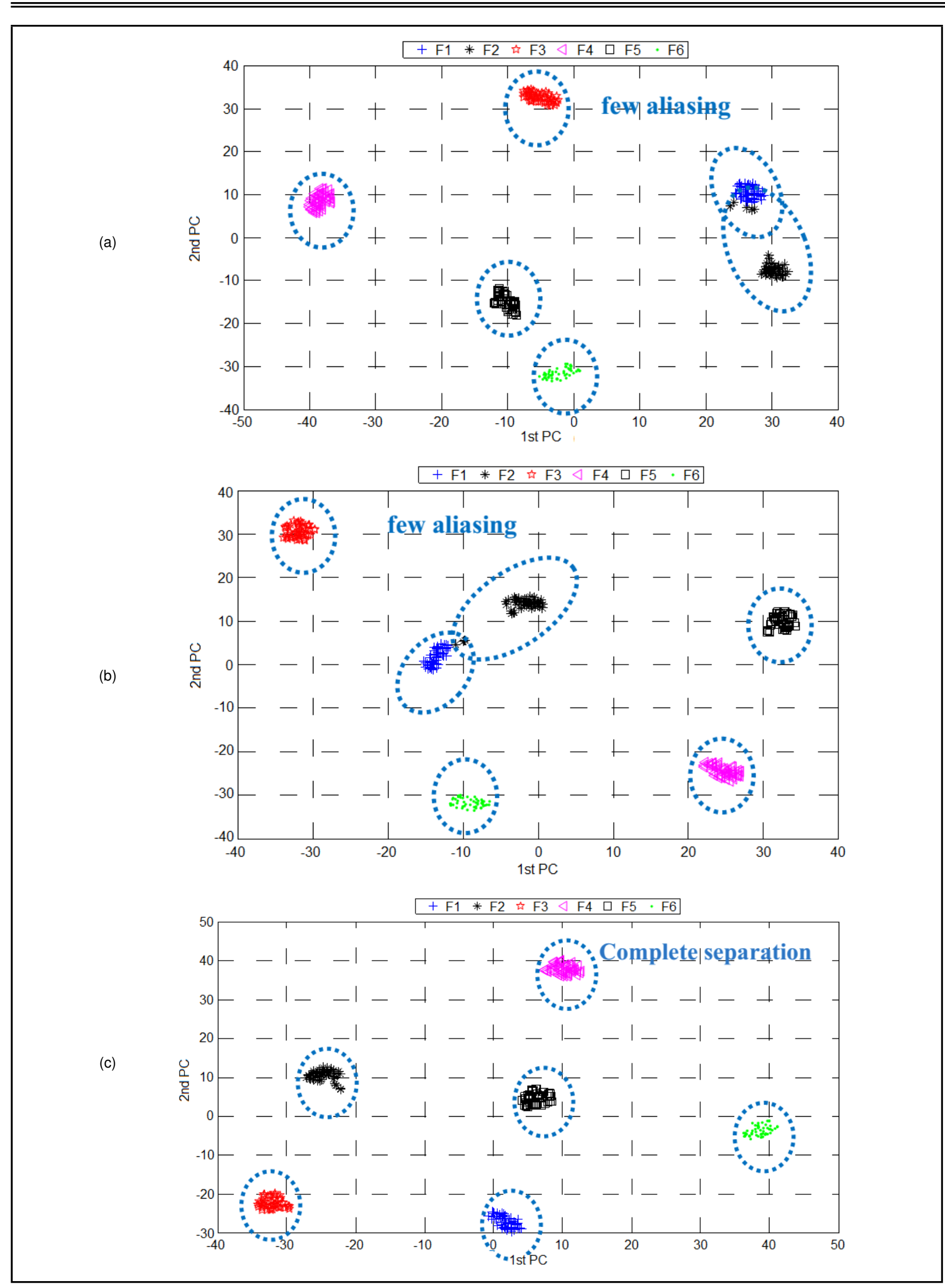

Figure 11. 2-dimensional feature visualization of testing samples a) OS-ISAE based-diagnostic model; b) VMD-ISAE based-diagnostic model; c) ORVMDISAE based-diagnostic model. 


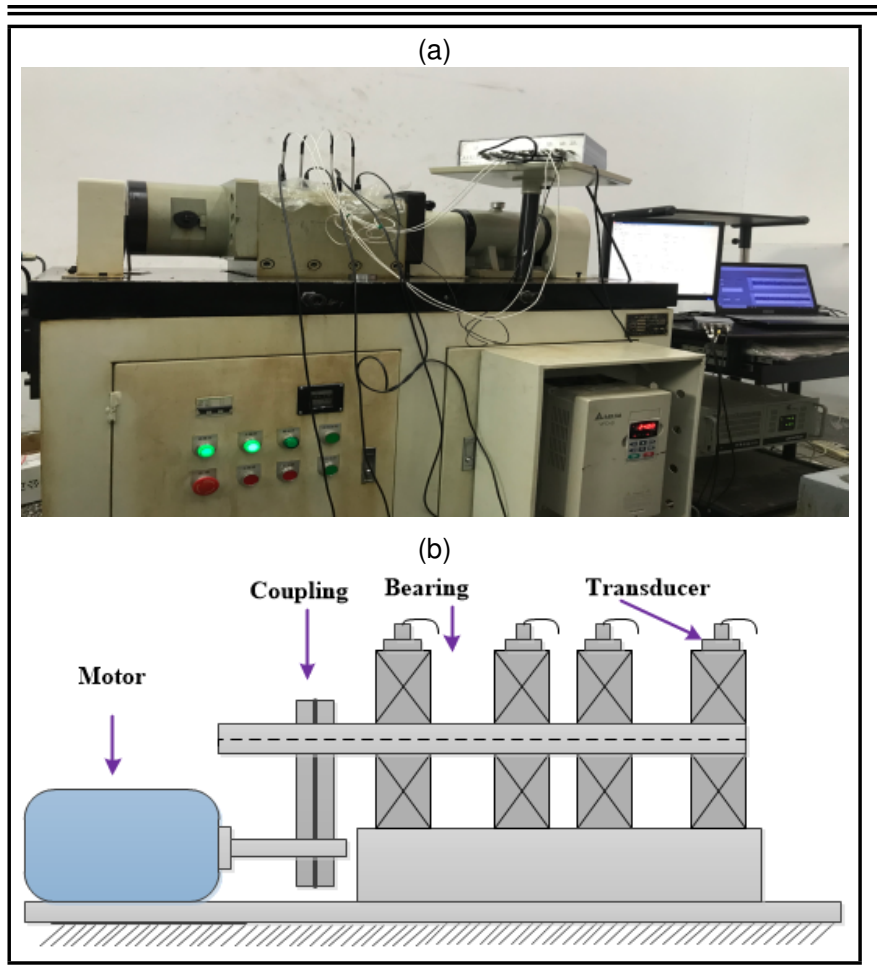

Figure 12. The illustration of ABLT-1A: a) the perspective view; b) the schematic diagram.

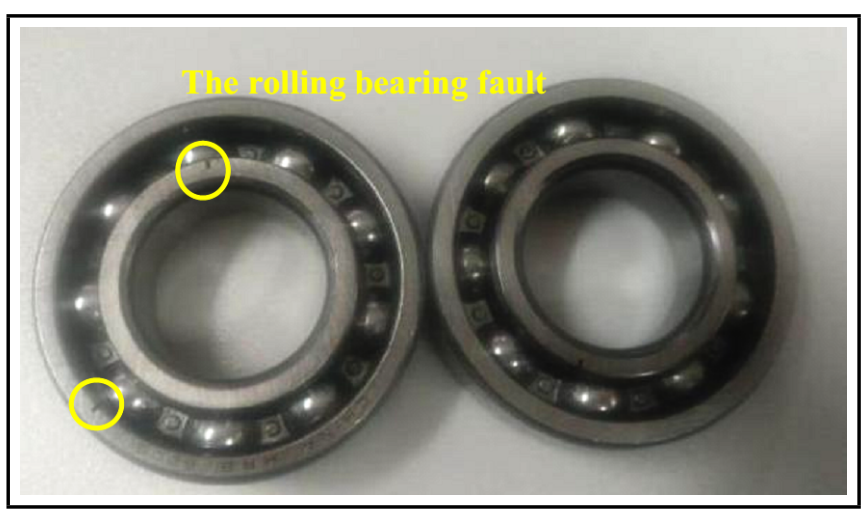

Figure 13. The failure view of the inner-outer ring compound fault.

Table 4. The experimental condition of ABLT-1A

\begin{tabular}{|c|c|}
\hline Experimental conditions & Specific parameters \\
\hline Bearing type & HRB6205 single row deep groove ball bearings \\
Number of test bearing & $4 \mathrm{sets}$ \\
Load & $0 \mathrm{~kg}$ \\
Data acquisition card & NI9234 \\
Sensor & PCB acceleration sensor \\
Sampling frequency & $10240 \mathrm{~Hz}$ \\
Rotating speed & $1050 \mathrm{r} / \mathrm{min}$ \\
Length of vibration signal & 102400 \\
\hline
\end{tabular}

resonance frequency, the envelope entropy of IMF4 was also the smallest. By envelope demodulation, the envelope spectrum is drawn in Fig. 17, in which the line amplitude of the spectrum was very prominent, which indicates that the characteristic frequency information was extracted. According to the concept of the index, relevant energy $(R e)$ index and relevant coefficient $(R c)$ between components and the raw signal was calculated in Table 5.

As illustrated in Table 5, the index $R e$ highlighted the correlation degree between different components and the original signal. The cumulative $R e$ index was equal to 0.8 , which can
Table 5. The $R e$ index of IMF component signals.

\begin{tabular}{||c|c|c||}
\hline Component signals & Relevant energy $(R e)$ & Relevant coefficient $(R c)$ \\
\hline IMF1 & 0.0661 & 0.3786 \\
IMF2 & 0.2255 & 0.6042 \\
IMF3 & 0.0335 & 0.3677 \\
IMF4 & 0.0157 & 0.3044 \\
IMF5 & 0.0140 & 0.2847 \\
IMF6 & 0.0158 & 0.2937 \\
IMF7 & 0.0370 & 0.3565 \\
IMF8 & 0.0343 & 0.3344 \\
\hline
\end{tabular}

fully cover the main information for signal reconstruction. The reconstructed signal is drawn in Fig. 18. After signal enhancement, the enhanced signal can be fed into deep learning for fault feature extraction and pattern recognition.

\subsubsection{Fault Classification and Recognition Results}

For experimental comparison, three kinds of signals, namely, the original signal after FFT transformation, VMD signal after FFT transformation and ORVMD after FFT transformation, were input into ISAE algorithm, respectively. The classification results are obtained in Fig. 19. Figure 19 indicates the fault confusion matrix of ABLT-1A rolling bearing fault data set based on ORVMD-ISAE, VMD-ISAE and OS-ISAE. The diagnosis results illustrated that the proposed framework can fully diagnose the five types of faults.

\subsubsection{Feature Visualization via t-SNE}

To validate that the proposed method can learn the effective feature representation from raw vibration signals, the extracted last layer of features via ISAE were visualized through the t-SNE. To remember the diagnosis model method \{original signal+ISAE; VMD+ISAE; ORVMD+ ISAE $\}$, t-SNE was used to convert the high dimensional representation into a 3dimensional graph. The above three diagnostic methods obtained the 3-dimensional scatter distribution diagram of characteristic points through t-SNE as shown in the Fig. 20. Only the ORVMD-SAE diagnostic model was able to divide the samples of various conditions, which indicated the effectiveness of the proposed framework.

\subsubsection{Compared with Other Diagnostic Methods}

Specifically, different combination fault diagnostic was selected as comparative testing to validate the effectiveness of the proposed method. The selected various combination methods were displayed as follows: \{ORVMD+ISAE $(R e)$, ORVMD+DBN $(R e), \mathrm{VMD}+\mathrm{SAE}(R e), \mathrm{VMD}+\mathrm{DBN}(R e)$, ORVMD+SAE $(R e)$, ORVMD+DBN $(R e)$, OVMD+DBN $(R c)$, OVMD+ISAE $(R c)=$ Method1, Method2, Method3, Method4, Method5, Method6, Method7, Method8\}. The combination fault diagnosis framework of cross-training and testing samples was adopted by changing the proportion of training samples and testing samples, ${ }^{46}$ each group was set to be $10 / 90,20 / 80,60 / 40,40 / 60,20 / 80$ and $10 / 90$, respectively. Afterwards, the average recognition accuracy of different methods is shown in Fig. 21.

Generally, the recognition accuracy of the above-mentioned eight methods were increased with the increasing of the number of training samples. Owing to the increasing of the number of training samples, the more prior information is obtained. It can be observed from Fig. 21 the proposed ORVMD-ISAE 


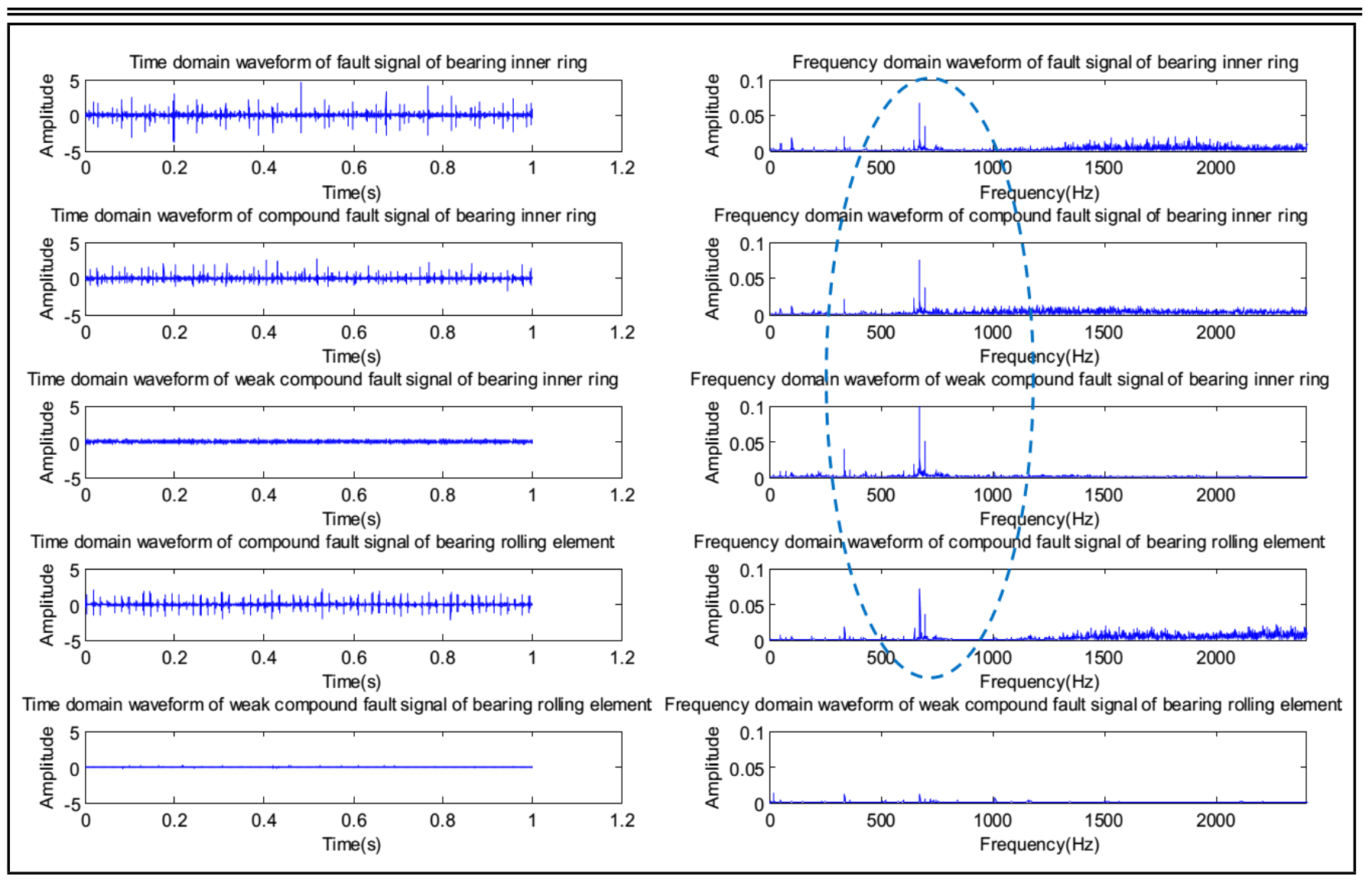

Figure 14. The waveform for different health condition of rolling bearing.

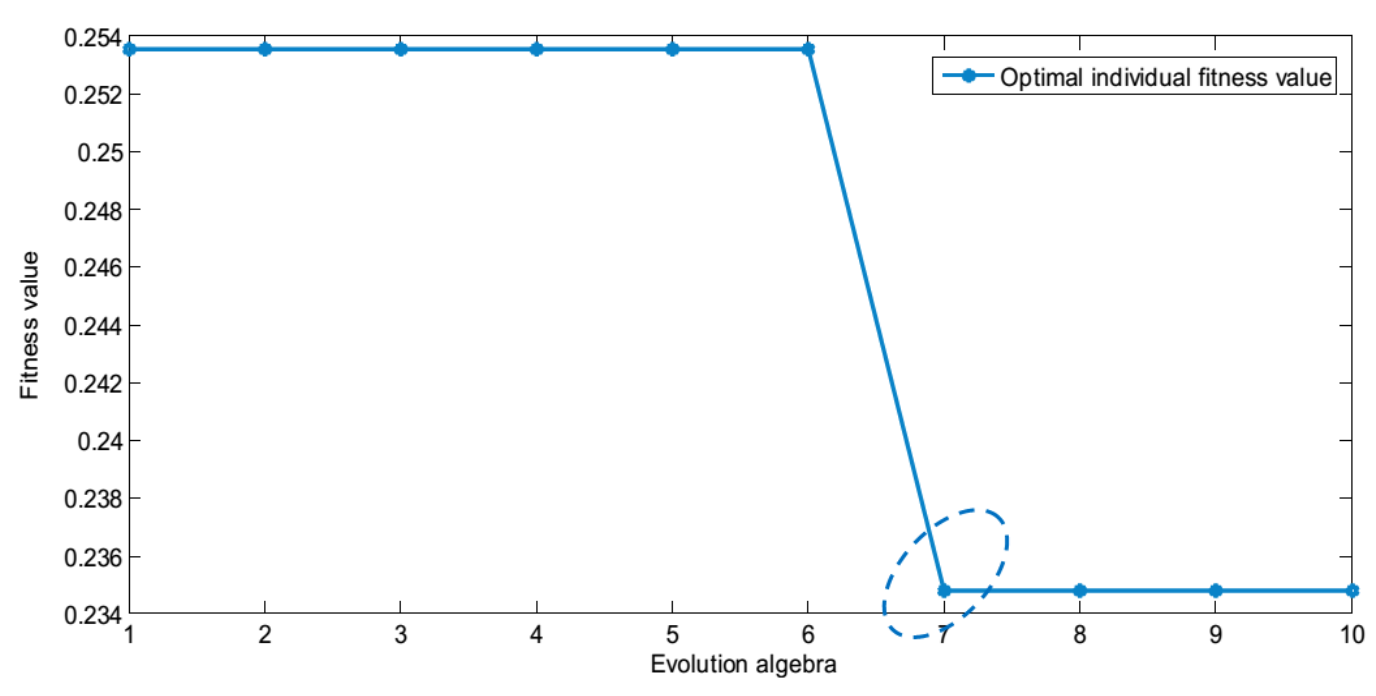

Figure 15. The minimum envelope entropy of inner ring weak fault signals (ABLT-1A) via ORVMD.

framework has good generalization performance. The proposed framework has the best anti-interference performance.

\section{CONCLUSIONS}

To improve the recognition accuracy of the weak fault diagnosis of rotating machinery, this paper proposes a new weak fault recognition framework. The core of the fault identification framework is to design the ORVMD algorithm for weak signal enhancement and the ISAE algorithm for weak fault identification. The designed ORVMD algorithm can comprehensively utilize the PSO and Re index to overcome the prob- lem of large error of conventional VMD in signal decomposition. At the same time, the designed ISAE algorithm can utilize the advantages of two types of regularization to improve the generalization performance of fault recognition. Finally, the experimental signals of the two types of rolling bearings validate the effectiveness of the proposed fault diagnosis framework and algorithm. The fault diagnosis framework proposed in this paper can provide a promising reference for the effective detection and diagnosis of weak faults in rotating machinery. In addition, we will use actual industrial field data to validate the effectiveness of the proposed fault diagnosis framework in future work. 


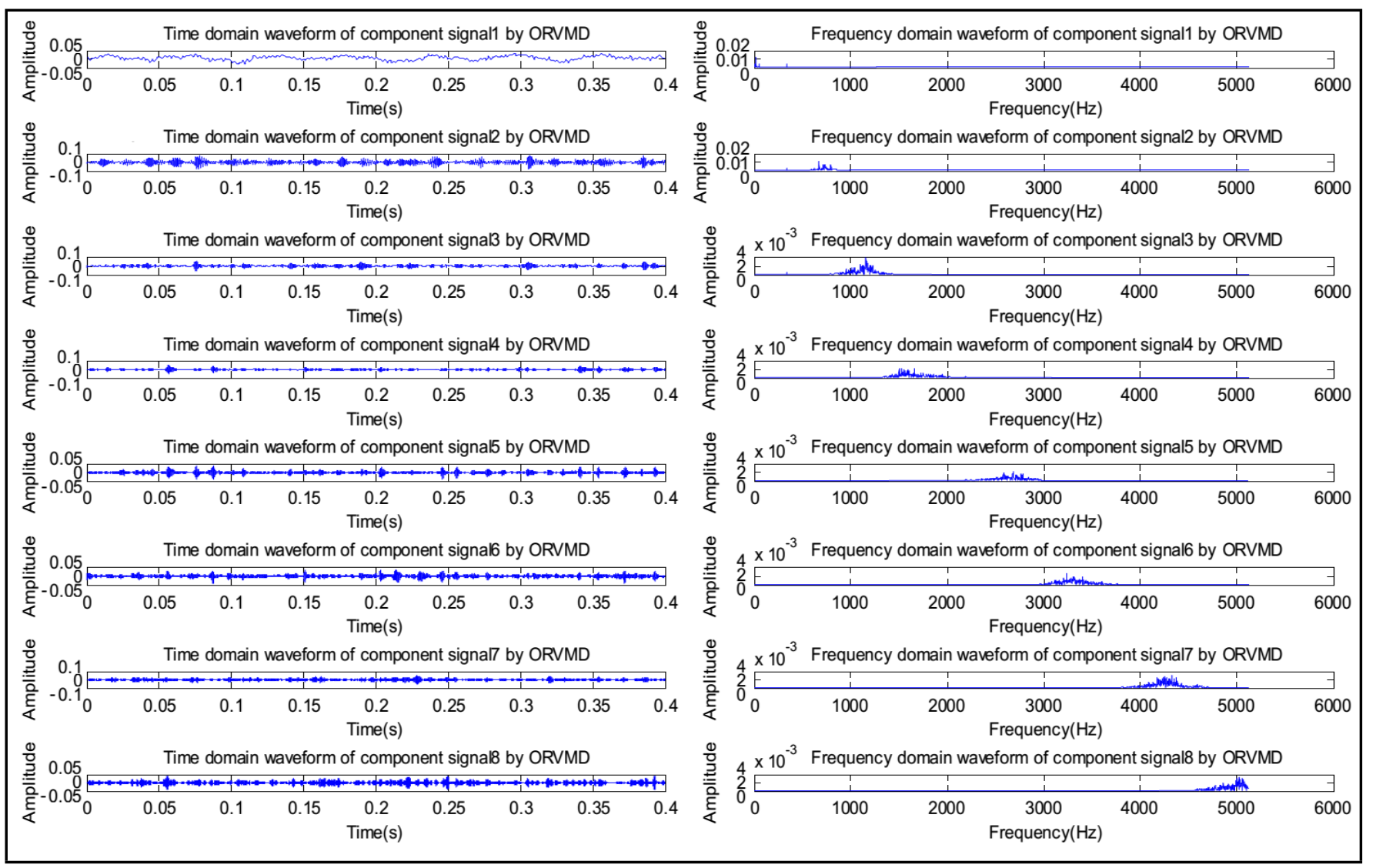

Figure 16. Time-domain and frequency-domain waveform for different IMF components.

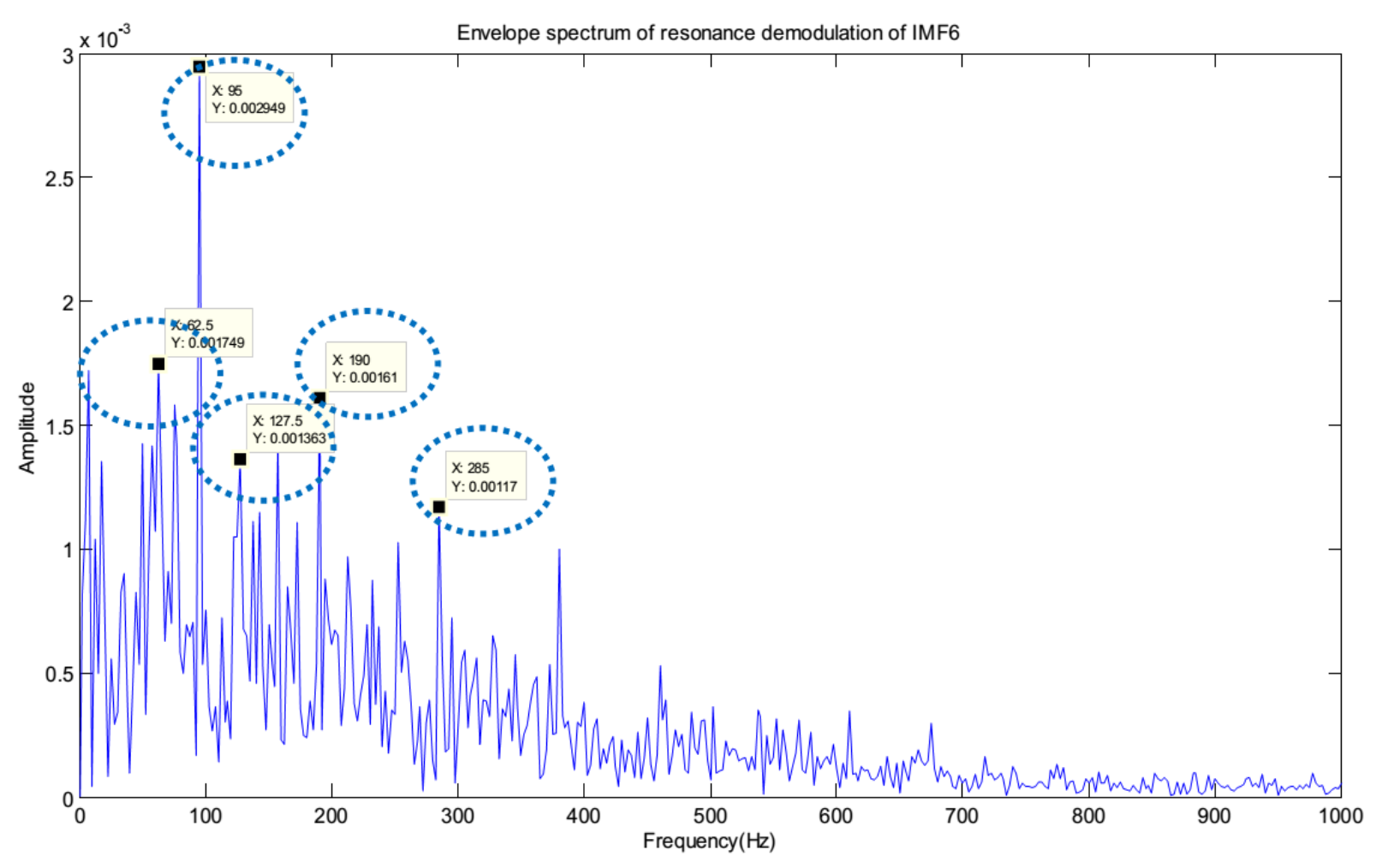

Figure 17. The envelope spectra of IMF6 from bearing inner-outer ring compound fault signal. 


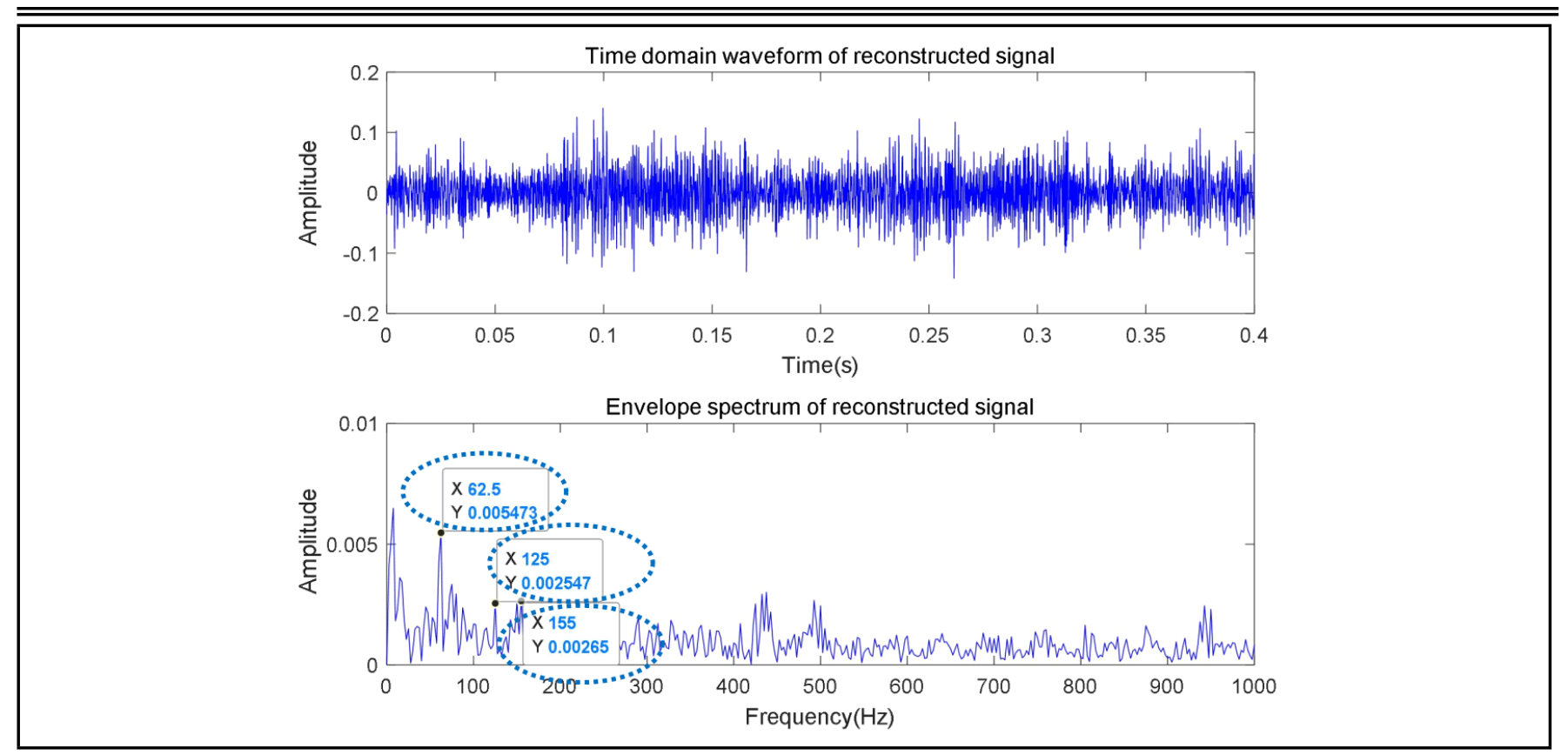

Figure 18. Time-frequency domain waveform and envelope spectrum of the reconstructed inner-outer ring weak fault signal.

\section{ACKNOWLEDGEMENTS}

This study first thanks to the bearing data provided by Case Western Reserve University. And the study also was funded by the National Natural Science Foundation of China (Grant No. 51675098), Postgraduate Research \& Practice Innovation Program of Jiangsu Province, China (No. SJKY19_0064) and Youth program of Jiangsu Natural Science Foundation (No. BK20190911). This research is also funded by China Scholarship Council (CSC) and the State Key Program of National Natural Science Foundation of China (No. 51937002).

\section{REFERENCES}

1 Shao, H., Cheng, J., Jiang, H., Yang, Y., and Wu, Z. Enhanced deep gated recurrent unit and complex wavelet packet energy moment entropy for early fault prognosis of bearing, Knowledge-Based Systems, 188, 105022, (2019). https://dx.doi.org/10.1016/j.knosys.2019.105022

2 Zhao, M., Kang, M., Tang, B., and Pecht, M. Deep residual networks with dynamically weighted wavelet coefficients for fault diagnosis of planetary gearboxes, IEEE Transactions on Industrial Electronics, 65 (5), 4290-4300, (2018). https://dx.doi.org/10.1109/TIE.2017.2762639

3 Yu, K., Lin, T. R., Ma, H., Li, H., and Zeng, J. A combined polynomial chirplet transform and synchroextracting technique for analyzing nonstationary signals of rotating machinery, IEEE Transactions on Instrumentation and Measurement, 69 (4), 1505-1518, (2020). https://dx.doi.org/10.1109/TIM.2019.2913058

4 Dong, S., Xu, X., and Luo, J. Mechanical fault diagnosis method based on LMD Shannon entropy and improved fuzzy C-means clustering, International Journal of Acoustics and Vibration, 22 (2), 211-217, (2017). https://dx.doi.org/10.20855/ijav.2017.22.2466
5 Zhang, J., Zhong, M., and Zhang, J. Detection for weak fault in planetary gear trains based on an improved maximum correlation kurtosis deconvolution, Measurement Science and Technology, 31 (2), 025101, (2019).

6 Zhang, F., Huang, J., Chu, F., and Cui, L. Mechanism and method for outer raceway defect localization of ball bearings, IEEE Access, 8, 4351-4360, (2020). https://dx.doi.org/10.1109/ACCESS.2019.2950284

7 He, Z., Shao, H., Zhang, X., Cheng, J., and Yang, Y. Improved deep transfer auto-encoder for fault diagnosis of gearbox under variable working conditions with small training samples, IEEE Access, 7, 115368-115377, (2019). https://dx.doi.org/10.1109/ACCESS.2019.2936243

8 Cheng, J., Yang, Y., Li, X., Pan, H., and Cheng, J. An early fault diagnosis method of gear based on improved symplectic geometry mode decomposition, Measurement, 151, 107140, (2020). https://dx.doi.org/10.1016/j.measurement.2019.107140

$9 \mathrm{Yu}$, G. Lin, T. Wang, Z., and Li, Y. Timereassigned multisynchrosqueezing transform for bearing fault diagnosis of rotating machinery, IEEE Transactions on Industrial Electronics, (2020). https://dx.doi.org/10.1109/TIE.2020.2970571

10 Yu, K., Ma, H., Han, H., Zeng, J., Li, H., Li, X., Xu, Z., and Wen, B. Second order multi-synchrosqueezing transform for rub-impact detection of rotor systems, Mechanism and Machine Theory, 140, 321-349, (2019). https://dx.doi.org/10.1016/j.mechmachtheory.2019.06.007

11 Huang, N. E., Shen, Z., Long, S. R., Wu, M. C., Shih, H. H., Zheng, Q., Yen, N.-C., Tung, C. C., and Liu, H. H. The empirical mode decomposition and the Hilbert spectrum for nonlinear and nonstationary 


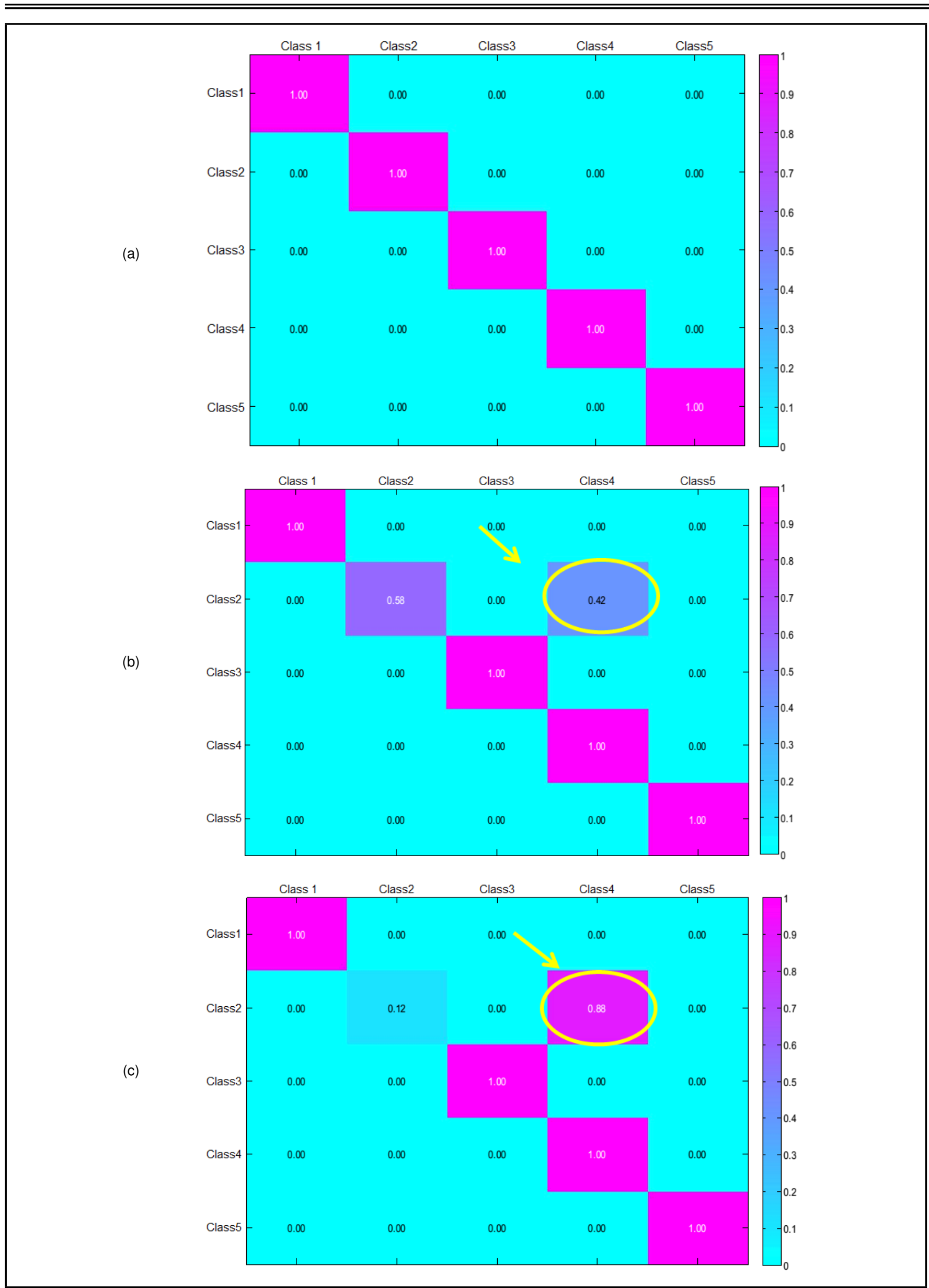

Figure 19. The confusion matrix for fault diagnosis based: a) ORVMD-ISAE; b) VMD-ISAE; c) OS-ISAE. 
(a)

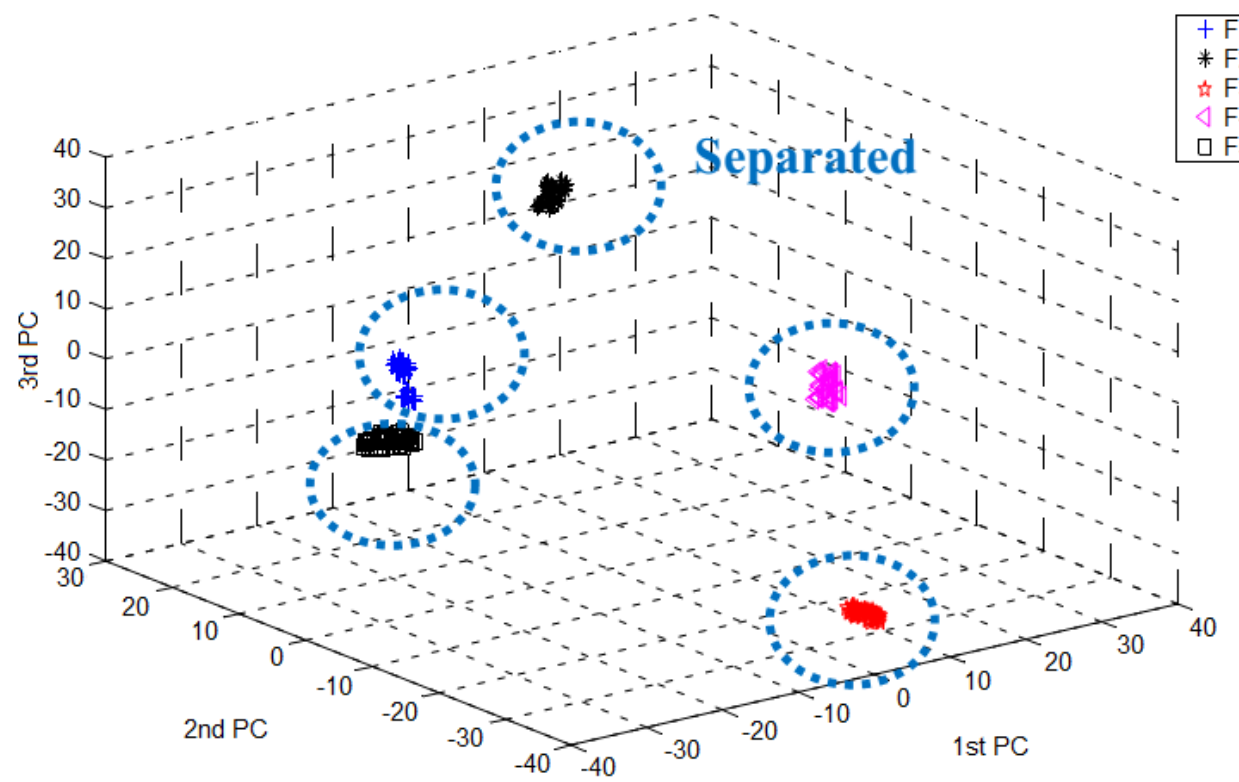

(b)

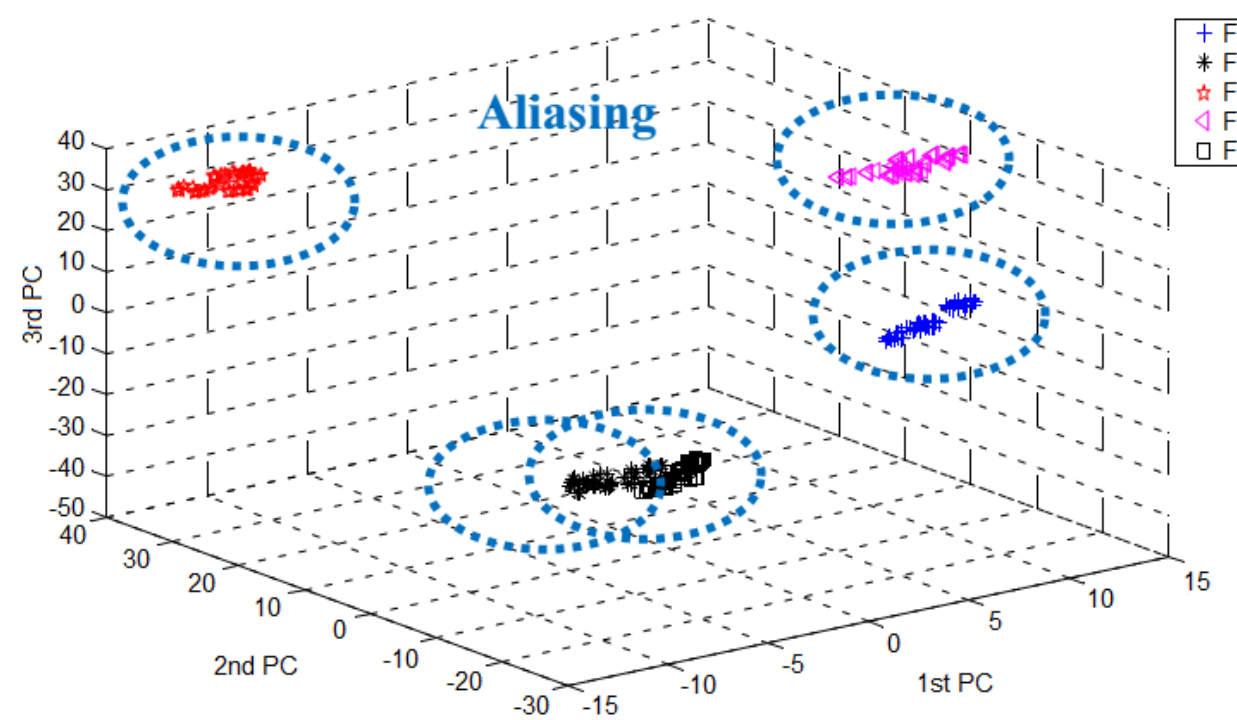

(c)

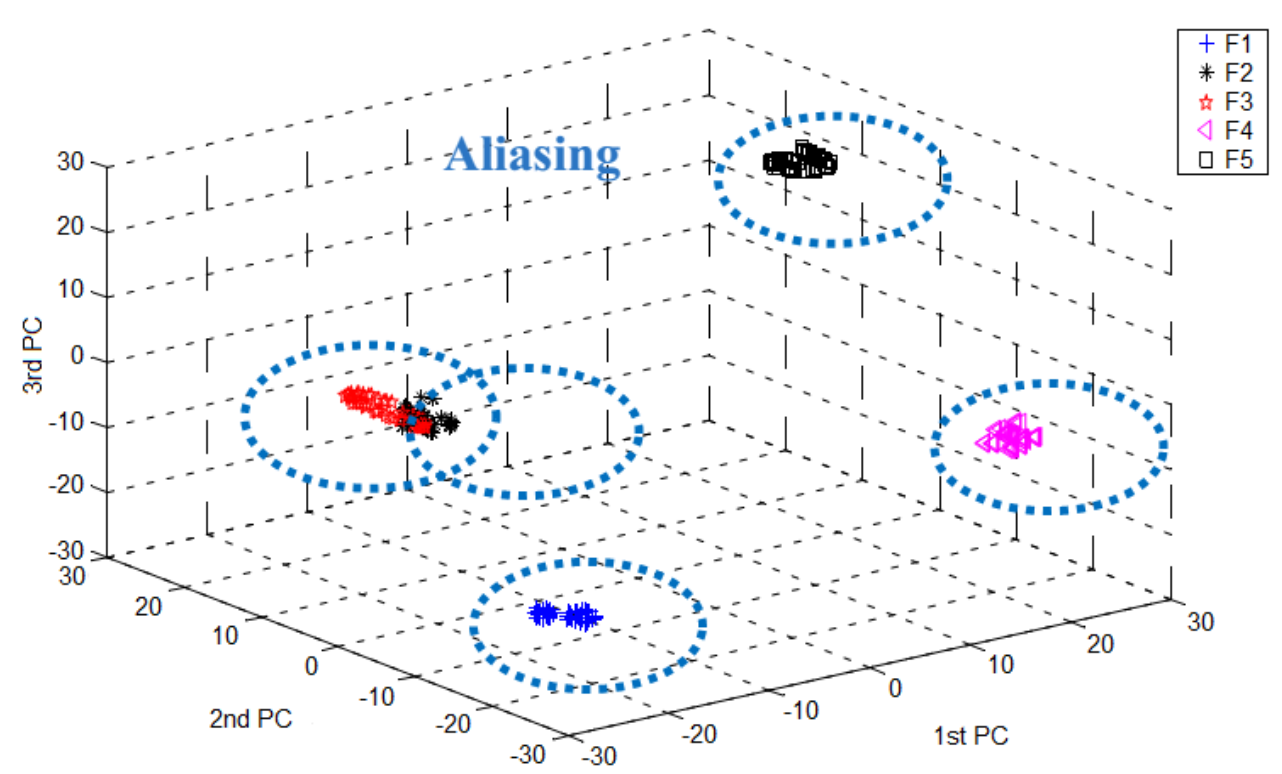

Figure 20. 3-dimensional feature visualization of testing samples based: a) ORVMD-ISAE; b) VMD-ISAE; c) OS-ISAE. 


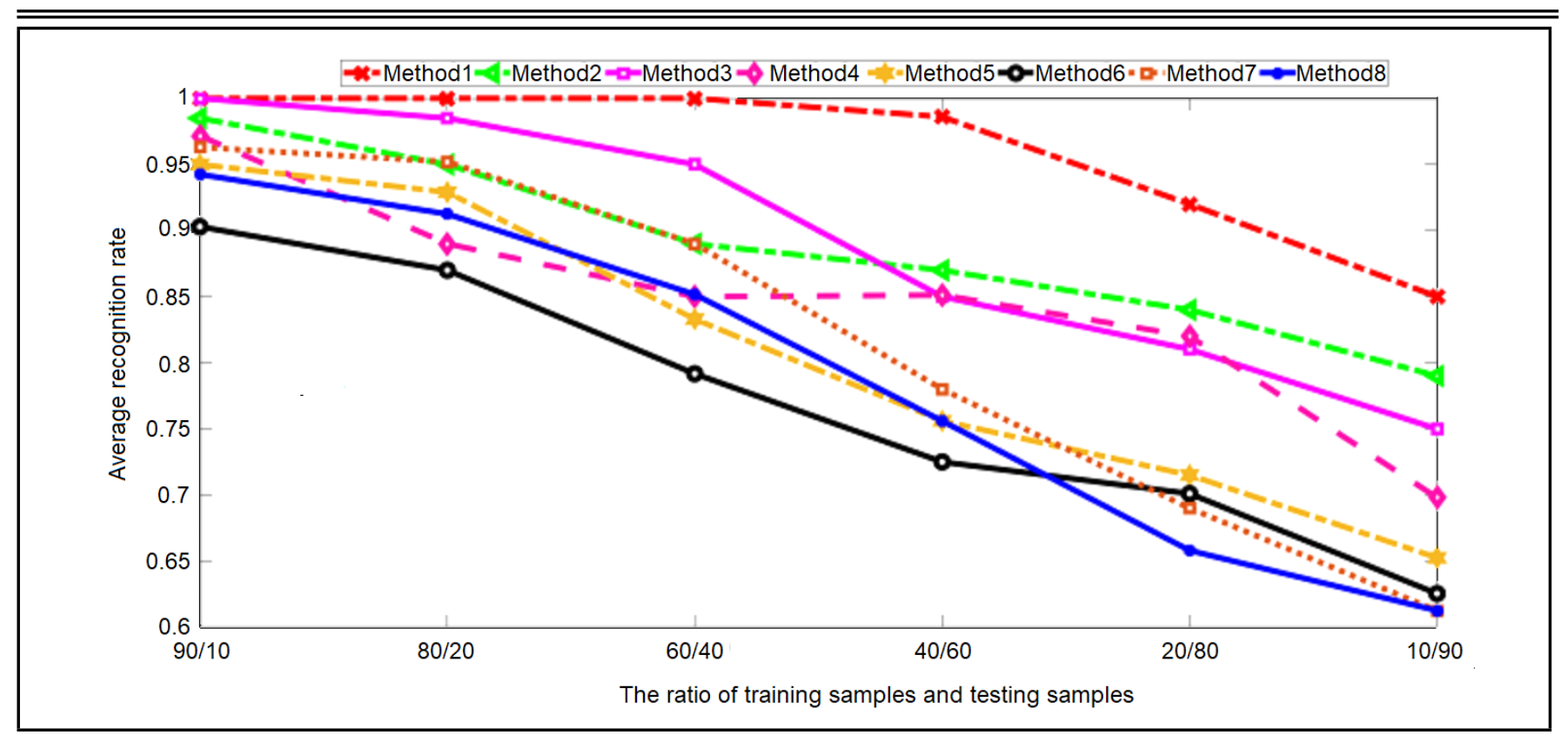

Figure 21. Average recognition accuracy of testing samples built on different training sample numbers.

time series analysis, Proceedings Mathematical Physical \& Engineering Sciences, 454, 903-995, (1998). https://dx.doi.org/10.1098/rspa.1998.0193

$12 \mathrm{Li}, \mathrm{C}$. and de Oliveira, J. V. Advances in intelligent computing for diagnostics, prognostics, and system health management, Journal of Intelligent \& Fuzzy Systems, 34 (6), 33973401, (2018). https://dx.doi.org/10.3233/JIFS-169520

13 Yan, X. A., Jia, M. P., and Xiang, L. Compound fault diagnosis of rotating machinery based on OVMD and a 1.5-dimension envelope spectrum, Measurement Science and Technology, 27, 075002, (2016). https://dx.doi.org/10.1088/0957-0233/27/7/075002

14 Smith, J. S. The local mean decomposition and its application to EEG perception data, Journal of the Royal Society Interface, 2 (5), 443-454, (2005). https://dx.doi.org/10.1098/rsif.2005.0058

15 Su, W. S., Wang, F. T., Zhang, Z. X., Guo, Z. G., and Li, H. K. Application of EMD denoising and spectral kurtosis in early fault diagnosis of rolling element bearings, Journal of Vibration and Shock, 22 (1), 3537-3540, (2010).

16 Bin, G. F., Gao, J. J., Li, X. J., and Dhillon, B. S. Early fault diagnosis of rotating machinery based on wavelet packets-Empirical mode decomposition feature extraction and neural network, Mechanical Systems and Signal Processing, 27 (1), 696-711, (2012). https://dx.doi.org/10.1016/j.ymssp.2011.08.002

17 Li, Y., Liang, X., Yang, Y., Xu, M., and Huang, W. Early fault diagnosis of rotating machinery by combining differential rational spline-based LMD and $\mathrm{K}-\mathrm{L}$ divergence, IEEE Transactions on Instrumentation and Measurement, 66 (11), 3077-3090, (2017). https://dx.doi.org/10.1109/TIM.2017.2664599
18 Yan, X. A., Jia, M. P., and Zhao, Z. Z. A novel intelligent detection method for rolling bearing based on IVMD and instantaneous energy distributionpermutation entropy, Measurement, 130, 435-447, (2018). https://dx.doi.org/10.1016/j.measurement.2018.08.038

19 Pan, J., Chen, J., Zi, Y., Li, Y., and He, Z. Monocomponent feature extraction for mechanical fault diagnosis using modified empirical wavelet transform via datadriven adaptive Fourier spectrum segment, Mechanical Systems and Signal Processing, , 72-73, 160-183, (2016). https://dx.doi.org/10.1016/j.ymssp.2015.10.017

20 Yong, L., Rui, Y., and Song, G. Multivariate empirical mode decomposition and its application to fault diagnosis of rolling bearing, Mechanical Systems and Signal Processing, 81, 219-234, (2016). https://dx.doi.org/10.1016/j.ymssp.2016.03.010

21 Dragomiretskiy, K. and Zosso, D. Variational mode decomposition, IEEE Transactions on Signal Processing, 62, 531544, (2014). https://dx.doi.org/10.1109/TSP.2013.2288675

22 Wang, Y., Markert, R., Xiang, J., and Zheng, W. Research on variational mode decomposition and its application in detecting rub-impact fault of the rotor system, Mechanical Systems and Signal Processing, 60-61, 243-251, (2015). https://dx.doi.org/10.1016/j.ymssp.2015.02.020

23 Mohanty, S., Gupta, K. K., and Raju, K. S. Hurst based vibro-acoustic feature extraction of bearing using EMD and VMD, Measurement, 117, 200-220, (2018). https://dx.doi.org/10.1016/j.measurement.2017.12.012

24 Zhang, X., Liang, Y., Zhou, J., and Zang, Y. A novel bearing fault diagnosis model integrated permutation entropy, ensemble empirical mode decomposition and optimized SVM, Measurement, 69, 164-179, (2015). https://dx.doi.org/10.1016/j.measurement.2015.03.017 
${ }^{25}$ Mohanty, S. and Gupta, K. K. Bearing fault analysis using variational mode decomposition, Journal of Instrumentation Technology \& Innovations, 4 (2), 20-27, (2014).

${ }^{26}$ An, X. and Yang, J. Denoising of hydropower unit vibration signal based on variational mode decomposition and approximate entropy, Transactions of the Institute of Measurement and Control, 38, 282-292, (2015). https://dx.doi.org/10.1177/0142331215592064

27 Kennedy, J. and Eberhart, R. Particle swarm optimization, Proc. of ICNN'95 IEEE International Conference on Neural Networks, 4, 1942-1948, (2011). https://dx.doi.org/10.1109/ICNN.1995.488968

${ }^{28}$ Zhao, X. and Jia, M. Fault diagnosis of rolling bearing based on feature reduction with global-local margin Fisher analysis, Neurocomputing, 315, 447-464, (2018). https://dx.doi.org/10.1016/j.neucom.2018.07.038

${ }^{29}$ He, Z., Shao, H., Lin, J., Cheng, J., and Yang, Y. Transfer fault diagnosis of bearing installed in different machines using enhanced deep autoencoder, Measurement, 152, 107393, (2020). https://dx.doi.org/10.1016/j.measurement.2019.107393

30 Zhao, X. and Jia, M. A novel deep fuzzy clustering neural network model and its application in rolling bearing fault recognition, Measurement Science and Technology, 29, 125005, (2018). https://dx.doi.org/10.1088/1361$6501 / \mathrm{aae} 27 \mathrm{a}$

${ }^{31}$ Hinton, G. E. and Salakhutdinov, R. Reducing the dimensionality of data with neural networks, Science, 313, 504507, (2006). https://dx.doi.org/10.1126/science.1127647

${ }^{32}$ LeCun, Y., Bengio, Y., and Hinton, G. Deep learning, Nature, 521, 436-444, (2015). https://dx.doi.org/10.1038/nature14539

${ }^{33}$ Zhao, X. and Jia, M. A new local-global deep neural network and its application in rotating machinery fault diagnosis, Neurocomputing, 366 (13), 215-233, (2019). https://dx.doi.org/10.1016/j.neucom.2019.08.010

34 Vincent, P., Larochelle, H., Lajoie, I., Bengio, Y., and Manzagol, P.-A. Stacked denoising autoencoders: learning useful representations in a deep network with a local denoising criterion, Journal of Machine Learning Research, 11 (12), 3371-3408, (2010).

35 Sun, W., Shao, S., Zhao, R., Yan, R., Zhang, X., and Chen, X. A sparse auto-encoder-based deep neural network approach for induction motor faults classification, Measurement, 89, 171-178, (2016). https://dx.doi.org/10.1016/j.measurement.2016.04.007

36 Junbo, T., Weining, L., Juneng, A., and Xueqian, W. Fault diagnosis method study in roller bearing based on wavelet transform and stacked auto-encoder, Proc. of the 27th Chinese Control and Decision Conference, 4608-4613, (2015). https://dx.doi.org/10.1109/CCDC.2015.7162738
37 Jia, X., Zhao, M., Di, Y., Li, P., and Lee, J. Sparse filtering with the generalized lp/lq norm and its applications to the condition monitoring of rotating machinery, Mechanical Systems and Signal Processing, 102, 198-213, (2018). https://dx.doi.org/10.1016/j.ymssp.2017.09.018

${ }^{38}$ Zhao, X. and Jia, M. A novel unsupervised deep learningnetwork for intelligent fault diagnosisof rotating machinery, Structural Health Monitoring, (2019). https://dx.doi.org/10.1177/1475921719897317

39 Brown, A. Universal development and L1-L2 convergence in bilingual construal of manner in speech and gesture in Mandarin, Japanese, and English, The Modern Language Journal, 99, 66-82, (2015). https://dx.doi.org/10.1111/j.1540-4781.2015.12179.x

${ }^{40}$ Ngiam, J., Chen, Z., Bhaskar, S. A., Koh, P. W., and Ng, A. Y. Sparse filtering, Proc. of Neural Information Processing Systems Conference NIPS 2011-Advances in Neural Information Processing Systems 24, 1125-1133, (2011).

${ }^{41}$ Chenglin, Z., Xuebin, S., Songlin, S., and Ting, J. Fault diagnosis of sensor by chaos particle swarm optimization algorithm and support vector machine, Expert Systems with Applications, 38 (8), 9908-9912, (2011). https://dx.doi.org/10.1016/j.eswa.2011.02.043

42 Xiong, J., Liang, Q., Wan, J., Zhang, Q., Chen, X., and Ma, $R$. The order statistics correlation coefficient and PPMCC fuse non-dimension in fault diagnosis of rotating petrochemical unit, IEEE Sensors Journal, 18 (11), 4704-4714, (2018). https://dx.doi.org/10.1109/JSEN.2018.2820170

${ }^{43}$ Loparo, K. A. Bearing data center, Case Western Reserve University, http://csegroups.case.edu/bearingdatacenter/home, (Accessed June 25, 2018).

44 Zhao, X., Jia, M., and Lin, M. Deep Laplacian autoencoder and its application into imbalanced fault diagnosis of rotating machinery, Measurement, 152, (2020). https://dx.doi.org/10.1016/j.measurement.2019.107320

45 Van der Maaten, L. and Hinton, G. Visualizing data using t-SNE, Journal of Machine Learning Research, 9, 25792605, (2008).

${ }^{46}$ Chen, F., Tang, B., and Chen, R. A novel fault diagnosis model for gearbox based on wavelet support vector machine with immune genetic algorithm, Measurement, 46 (1), 220-232, (2013). https://dx.doi.org/10.1016/j.measurement.2012.06.009 